\title{
REPRESENTATIONS OF THE FREE PROFINITE OBJECT OVER DA
}

\author{
ANA MOURA
}

\begin{abstract}
In this paper, we extend to DA some techniques developed by Almeida and Weil, and Almeida and Zeitoun for the pseudovariety $\mathrm{R}$ to obtain representations of the implicit operations on DA: by labeled trees of finite height, by quasi-ternary labeled trees, and by labeled linear orderings. We prove that two implicit operations are equal over DA if and only if they have the same representation, for any of the three representations. We end the paper by relating these representations.
\end{abstract}

\section{INTRODUCTION}

The importance of the study of pseudovarieties of finite monoids became evident with Eilenberg [10] in the middle of the 1970's, who established the correspondence between varieties of rational languages and those classes of finite monoids. Some years later, Reiterman [11] showed that every pseudovariety of finite monoids is defined by some set of finitary pseudoidentities, which are equalities between implicit operations. As implicit operations over a pseudovariety of monoids contain information on the structure of the finite monoids in the pseudovariety, it became important to develop the study of the set of implicit operations over a pseudovariety $\mathrm{V}$ on a finite alphabet $A, \bar{\Omega}_{A} \mathrm{~V}$, which has the structure of a pro- $\mathrm{V}$ monoid.

Schützenberger [13] noted the interest of the study of the pseudovariety DS and Almeida and Weil [7] stated that for this pseudovariety and its subpseudovarieties it should be easy to make a description of the free profinite object. In fact, Almeida [1] factorized each element of the free profinite monoid over $\mathrm{J}$ in terms of component projections and idempotents and Azevedo [9] proved that a similar kind of factorization could be implemented to any subpseudovariety of DS, although it has not yet been discovered a canonical form of such factorizations in this pseudovariety. Almeida and Weil [7] gave two complementary descriptions of the monoid of implicit operations on $R$, one by labeled ordinals and the other by labeled infinite trees of finite depth. They did a similar study for the pseudovariety DRG. On the other hand, in their recent work, Almeida, Costa and Zeitoun [5,6] presented structural properties of the free profinite semigroup over A.

In their "Diamonds are forever", Tesson and Thérien [14] showed that languages whose syntactic monoid lies in DA have powerful characterizations, from combinatorial ones, to logical and even automata-theoretical ones. This characterizations

2000 Mathematics Subject Classification. 20M05; 20M07; $20 \mathrm{M} 35$.

Key words and phrases. Finite monoid; pseudovariety; profinite monoid; implicit operation; aperiodic; regular D-class. 
are useful to solve problems in computational and complexity theory and the authors gave examples of such applications. They emphasize that these problems are efficiently solvable when the syntactic monoid, besides being aperiodic, is in DA.

Thus, it becomes interesting to characterize the free profinite object over DA. In this paper, we present three representations of the free pro-DA object on a finite alphabet extending techniques developed for the pseudovariety R: the first one by means of finite-height labeled trees, using the ideas of Almeida and Weil [7], the second one by means of quasi-ternary labeled trees, based on the work of Almeida and Zeitoun [8] and the last one by means of labeled linear orderings, extending the description done by Almeida and Weil [7]. In connection with the second representation, we also exhibit a representation by wrapped automata, which turns out to be useful for recent work of the author.

The paper is organized as follows. In Section 2, we recall the basics of the theory of pseudovarieties of monoids, pro- $\mathrm{V}$ monoids and some notions on automata and linear orderings. In Section 3, we use the central basic factorization of an implicit operation on DA and we present two forms of iteration of that factorization. We prove the convergence of the infinite product resulting from the iterated factorizations and we end it with a characterization of the idempotents in terms of the type of iterated central basic factorization. In Section 4, we present the representations of the implicit operations over this pseudovariety that we had announced above. We end the section by relating the various representations.

\section{Preliminaries}

We briefly recall some basics of the theory of pseudovarieties of monoids, profinite monoids, automata and linear orderings and we introduce some related notation. We recommend [2,4] for a better understanding of these concepts and [12] as a reference on linear orderings.

In this paper, $A$ is a finite set called alphabet and its elements are called letters. We denote by $A^{*}$ (respectively by $A^{+}$) the free monoid (respectively the free semigroup) generated by $A$, whose elements are called words. The empty word is denoted by 1 . The length of a word $u$ is denoted by $|u|$ and the cardinality of $A$ is denoted by $|A|$. The content of a word $u$ is the smallest subset $B$ of $A$ such that $u \in B^{*}$. In particular, the content of the empty word is $\emptyset$. Finally, a word $u=a_{1} \cdots a_{n}$, with $a_{i} \in A$, for all $i$, is a subword of $v$ if there exist words $v_{0}, v_{1}, \ldots, v_{n} \in A^{*}$ such that $v=v_{0} a_{1} v_{1} \cdots a_{n} v_{n}$.

Given a semigroup $S$, we denote by $S^{1}$ the monoid defined as follows: if $S$ is itself a monoid, then $S^{1}=S$; otherwise, $S^{1}=S \cup\{1\}$, where 1 is an element that does not belong to $S$ and the multiplication in $S^{1}$ is the (unique) extension to the multiplication in $S$ in which 1 acts as a neutral element. For $s \in S$, we denote by $s^{\omega}$ the unique idempotent in the subsemigroup generated by $s$ and we set $s^{\omega+1}=s^{\omega} s$.

A class of finite monoids that is closed under taking submonoids, homomorphic images and finite direct products is called a pseudovariety and generally denoted by $\mathrm{V}$. For example, $\mathrm{M}$ is the pseudovariety of all finite monoids, $\mathrm{R}$ is the pseudovariety of all $\mathcal{R}$-trivial monoids, where a monoid $S$ is $\mathcal{R}$-trivial if, for all $s, t \in S$, $s \mathcal{R} t$ implies $s=t$. In this paper, we are interested in DA, the pseudovariety of monoids whose regular $\mathcal{D}$-classes are aperiodic semigroups. Note that a semigroup $S$ is aperiodic if $s^{\omega}=s^{\omega+1}$, for all $s \in S$, and a monoid $S$ is in DA if and only if, for all $s, t \in S$, we have $(s t)^{\omega}(t s)^{\omega}(s t)^{\omega}=(s t)^{\omega}$ and $s^{\omega}=s^{\omega+1}$. 
A topological monoid is a monoid equipped with a topology for which the multiplication in the monoid is a continuous function. We view a finite monoid as a topological monoid with respect to the discrete topology. A topological monoid $S$ is a profinite monoid (respectively a pro- $\mathrm{V}$ monoid) if it is a compact monoid which is residually finite (respectively residually in $\mathrm{V}$ ), which means that, whenever $s, t \in S$ and $s \neq t$, there exists a continuous homomorphism $\varphi: S \rightarrow F$ into a finite monoid (respectively into a member of $\mathrm{V}$ ) such that $\varphi(s) \neq \varphi(t)$. It is well known that profinite monoids are 0-dimensional, which means that the topology has an open basis consisting of clopen sets (which is equivalent to being a totally disconnected monoid).

Given an alphabet $A$ and a pseudovariety $\mathrm{V}$, the free pro- $\mathrm{V}$ monoid on $A$, denoted by $\bar{\Omega}_{A} \mathrm{~V}$, is the unique (up to isomorphism of topological monoids) pro- $\mathrm{V}$ monoid such that, for every mapping $\mu: A \rightarrow T$ into a pro- $\mathrm{V}$ monoid $T$, there is a unique continuous homomorphism $\hat{\mu}: \bar{\Omega}_{A} \mathrm{~V} \rightarrow T$ such that $\hat{\mu} \circ \iota=\mu$, where $\iota: A \rightarrow \bar{\Omega}_{A} \mathrm{~V}$ is the natural generating function. The elements of $\bar{\Omega}_{A} \mathrm{~V}$ are called implicit operations on $\mathrm{V}$ or pseudowords. For a pseudovariety $\mathrm{V}$ containing $\mathrm{SI}$, the content function is the unique continuous homomorphism $c: \bar{\Omega}_{A} \mathrm{~V} \rightarrow \mathcal{P}(A)$ such that $c \iota(a)=\{a\}$, for all $a \in A$.

A pseudoidentity is an equality of the form $u=v$, with $u, v \in \bar{\Omega}_{A} \mathrm{M}$, and $|A|$ is called the arity of the pseudoidentity. We say that a pseudoidentity is valid in a profinite monoid $T$, and we write $T \models u=v$, if $\varphi(u)=\varphi(v)$ for every continuous homomorphism $\varphi: \bar{\Omega}_{A} \mathrm{M} \rightarrow T$. Reiterman's Theorem [11] says that every pseudovariety is defined by some set of finitary ( $A$ is finite) pseudoidentities. That the class of all finite monoids which verify all the elements of a set of pseudoidentities is a pseudovariety follows immediately from the fact that the validity of a pseudoidentity in a finite monoid is preserved under taking homomorphic images, submonoids and finite direct products. For example, the pseudovariety DA is defined by the set of pseudoidentities $\left\{(x y)^{\omega}(y x)^{\omega}(x y)^{\omega}=(y x)^{\omega}, x^{\omega}=x^{\omega+1}\right\}$.

A deterministic automaton over an alphabet $A$ is a tuple $\mathcal{A}=(V, \rightarrow, q, F)$, where $V$ is the set of states, $q \in V$ is the initial state, $F \subseteq V$ is the set of final states and $\rightarrow: V \times A \rightarrow V$ is its transition function. We denote by $v . a$ the state reached from $v$ by reading the letter $a$, if this state exists, and we denote by $v . L$ the set of states reached from $v$ by reading some word of $L$.

Finally, we suppose that the reader is acquainted with the basic notions of linear orderings. In this paper, we use two different linear orderings of the set of natural numbers: the usual ordering, $R_{\mathbb{N}}$, and the backwards ordering, $R_{\mathbb{N}}^{*}$. We also use suborderings of these orderings and operations on linear orderings. We denote by $\omega, \omega^{*}$ and $\mathbf{n}$ the order type of $\left\langle\mathbb{N}, R_{\mathbb{N}}\right\rangle,\left\langle\mathbb{N}, R_{\mathbb{N}}^{*}\right\rangle$ and $\langle P, R\rangle$, which is a subordering of $\left\langle\mathbb{N}, R_{\mathbb{N}}\right\rangle$ with $|P|=n$, respectively.

\section{FACTORIZATION OF IMPLICIT OPERATIONS AND CONVERGENCE OF INFINITE PRODUCTS IN PRO-DA MONOIDS}

Let $w \in \bar{\Omega}_{A} \mathrm{DA} \backslash\{1\}$. We define the central basic factorization of $w$ (see Almeida [3] or Trotter and Weil [15]) as a factorization of one of the following forms:

(i) standard form: $w=\alpha a \gamma b \beta$ with $a, b \in A, \alpha, \beta, \gamma \in \bar{\Omega}_{A} \mathrm{DA}, a \notin c(\alpha)$, $b \notin c(\beta)$ and $c(\alpha a)=c(b \beta)=c(w)$;

(ii) overlapped form: $w=\alpha b \gamma a \beta$ with $a, b \in A, \alpha, \beta, \gamma \in \bar{\Omega}_{A} \mathrm{DA}, a \notin c(\alpha b \gamma)$, $b \notin c(\gamma a \beta)$ and $c(\alpha b \gamma a)=c(b \gamma a \beta)=c(w)$; 
(iii) degenerate form: $w=\alpha a \beta$ with $a \in A, \alpha, \beta \in \bar{\Omega}_{A} \mathrm{DA}, a \notin c(\alpha), a \notin c(\beta)$ and $c(\alpha a)=c(a \beta)=c(w)$.

By the following theorem, the central basic factorization of $w \in \bar{\Omega}_{A} \mathrm{DA} \backslash\{1\}$ exists and is unique:

Proposition 3.1 (Almeida [3]). Let $w \in \bar{\Omega}_{A} \mathrm{DA} \backslash\{1\}$. Then $w$ has a unique central basic factorization. In other words, if $w, w^{\prime} \in \bar{\Omega}_{A} \mathrm{DA} \backslash\{1\}$, the equality $w=w^{\prime}$ is valid in DA, and $\Phi$ and $\Psi$ are central basic factorizations of $w$ and $w^{\prime}$, respectively, then the two factorizations are both of the same type ((i),(ii) or (iii)) and the equalities of factors in corresponding positions are valid in DA.

We denote the central basic factorization of $w$ by the tuple $\operatorname{CBF}(w)=(\alpha, a, \gamma, b, \beta)$ or by the triple $\operatorname{CBF}(w)=(\alpha, a, \beta)$, as it is of the standard or overlapped form, or of the degenerate form, respectively.

For what follows, we define two different types of iteration of this factorization: in the first one we iterate the factorization while the content of the central factor (if it exists) does not decrease and in the second one we iterate until the central factor (if it exists) becomes 1 . We proceed to explain this in detail.

Let $\gamma_{0}=w$. If $c\left(\gamma_{k}\right)=c(w)$, we consider the central basic factorization of $\gamma_{k}$ which, in the case of being of the standard form, is $\gamma_{k}=\alpha_{k+1} a_{k+1} \gamma_{k+1} b_{k+1} \beta_{k+1}$. The $(k+1)$-iteration of the central basic factorization of $w$ is $w=\alpha_{1} a_{1} \cdots \alpha_{k+1}$. $a_{k+1} \gamma_{k+1} b_{k+1} \beta_{k+1} \cdots b_{1} \beta_{1}$ and $\gamma_{k+1}$ is called the remainder of order $k+1$. We iterate this process while $\gamma_{k}$ exists and $c\left(\gamma_{k}\right)=c(w)$. If, for any $n, c\left(\gamma_{n}\right) \neq c(w)$ and $\gamma_{n-1}$ admits a central basic factorization of the standard form, then $w=$ $\alpha_{1} a_{1} \cdots \alpha_{n} a_{n} \gamma_{n} b_{n} \beta_{n} \cdots b_{1} \beta_{1}$ is called the iterated central basic factorization of type 1 of $w$ and is called standard and of length $n$. If $c\left(\gamma_{n-1}\right)=c(w)$ and $\gamma_{n-1}$ has an overlapped central basic factorization, then $w=\alpha_{1} a_{1} \cdots \alpha_{n} b_{n} \gamma_{n} a_{n} \beta_{n} \cdots b_{1} \beta_{1}$ is the iterated central basic factorization of type 1 of $w$ and is called overlapped and of length $n$. If $c\left(\gamma_{n-1}\right)=c(w)$ and $\gamma_{n-1}$ has a degenerate central basic factorization, $\gamma_{n-1}=\alpha_{n} a_{n} \beta_{n}$, then $w=\alpha_{1} a_{1} \cdots \alpha_{n} a_{n} \beta_{n} \cdots b_{1} \beta_{1}$ is the iterated central basic factorization of type 1 of $w$ and is called degenerate and of length $n$. We say that, in the first two cases, $\gamma_{n}$ is the remainder of the central basic factorization of $w$, while in the degenerate case there is no remainder. Finally, if $c\left(\gamma_{n}\right)=c(w)$, for all $n$, we say that $w$ admits an infinite iterated central basic factorization of type 1 and we write $w=\alpha_{1} a_{1} \alpha_{2} a_{2} \cdots \cdots b_{2} \beta_{2} b_{1} \beta_{1}$. We denote the iterated central basic factorization of type 1 by $\mathrm{I}_{1} \operatorname{CBF}(w)$. Note that all the factors involved in this factorization have content strictly contained in $c(w)$.

Now, let $w=\alpha_{1} a_{1} \gamma_{1} b_{1} \beta_{1}$ be the central basic factorization of $w$. While $\gamma_{k} \neq 1$ or $\gamma_{k-1}$ does not admit a degenerate central basic factorization, we consider the central basic factorization of $\gamma_{k}$. The iterated central basic factorization of type 2 of $w$, $\mathrm{I}_{2} \mathrm{CBF}(w)$, is defined by one of the following forms: $w=\alpha_{1} a_{1} \alpha_{2} a_{2} \cdots \alpha_{n} a_{n} \beta_{n} \cdots b_{2}$. $\beta_{2} b_{1} \beta_{1}$, in case $\operatorname{CBF}\left(\gamma_{n-1}\right)$ is degenerate, $w=\alpha_{1} a_{1} \alpha_{2} a_{2} \cdots \alpha_{n} a_{n} b_{n} \beta_{n} \cdots b_{2} \beta_{2} b_{1} \beta_{1}$, in case $\gamma_{n}=1$, or $w=\alpha_{1} a_{1} \alpha_{2} a_{2} \cdots \cdots b_{2} \beta_{2} \cdot b_{1} \beta_{1}$, if the iteration is infinite. Note that, also in this iterated basic factorization, all the factors involved have content strictly contained in $c(w)$.

Results from Almeida [3, 2] and Almeida and Weil [7] allow us to conclude that we can iterate the central basic factorization of any of these two types and that the infinite product, in fact, converges. 
Lemma 3.2 (Almeida and Weil [7]). Let $S$ be a compact monoid. Then any two accumulation points of every right infinite product in $S$ are $\mathcal{R}$-equivalent.

Lemma 3.3 (dual of the previous lemma). Let $S$ be a compact monoid. Then any two accumulation points of every left infinite product in $S$ are $\mathcal{L}$-equivalent.

Corollary 3.4. Let $S$ be a pro-DA monoid. Given a right infinite product in $S$ and a left infinite product in $S$ such that their accumulation points are in the same regular $\mathcal{J}$-class, then the product of any right accumulation point by any left accumulation point is independent of the choice of these points.

Proof. It is enough to observe that the product of any two accumulation points of each of the two infinite products is in the regular $\mathcal{H}$-class $R \cap L$ which is trivial, where $R$ is the regular $\mathcal{R}$-class that contains all the accumulation points of the right infinite product and $L$ is the regular $\mathcal{L}$-class that contains all the accumulation points of the left infinite product.

We denote by $\vec{\prod}_{k=1}^{n} u_{k}$ the product $u_{1} u_{2} \cdots u_{n}$ and by $\overleftarrow{\prod}_{k=1}^{n} v_{k}$ the product $v_{n} \cdots v_{2} v_{1}$. Given a pro-DA monoid $S$ and sequences $\left(u_{k}\right)_{k \geq 1},\left(v_{k}\right)_{k \geq 1} \in S^{\mathbb{N}}$ in the conditions of the previous corollary, we denote by $\vec{\prod}_{k \geq 1} u_{k} \cdot \overleftarrow{\prod}_{k \geq 1} v_{k}$ the product of an accumulation point of the sequence $\left(\vec{\prod}_{k=1}^{n} u_{k}\right)_{n}$ by an accumulation point of the sequence $\left(\overleftarrow{\prod}_{k=1}^{n} v_{k}\right)_{n}$, when $n$ goes to infinity.

Therefore, given $w \in \bar{\Omega}_{A} \mathrm{DA} \backslash\{1\}$, the iterated central basic factorization of $w$ is of one of the following forms:

$$
w=\vec{\prod}_{k=1}^{n}\left(\alpha_{k} a_{k}\right) \cdot \gamma_{n} \cdot \overleftarrow{\prod}_{k=1}^{n}\left(b_{k} \beta_{k}\right)
$$

if it is of type 1 and it is finite and of the standard form or of the overlapped form, or

$$
w=\vec{\prod}_{k=1}^{n}\left(\alpha_{k} a_{k}\right) \cdot \overleftarrow{\prod}_{k=1}^{n}\left(b_{k} \beta_{k}\right)
$$

if is of type 2 and it is finite and $\operatorname{CBF}\left(\gamma_{n-1}\right)$ is of the standard form or of the overlapped form, or

$$
w=\vec{\prod}_{k=1}^{n}\left(\alpha_{k} a_{k}\right) \cdot \beta_{n} \cdot \overleftarrow{\prod}_{k=1}^{n-1}\left(b_{k} \beta_{k}\right)
$$

if is of type 1 or 2 and it is finite and degenerate, or

$$
w=\vec{\prod}_{k \geq 1}\left(\alpha_{k} a_{k}\right) \cdot \overleftarrow{\prod}_{k \geq 1}\left(b_{k} \beta_{k}\right)
$$

if the iteration is infinite (and of any type). In fact, the last equality is valid as we see from Lemma 3.9 or from Lemma 3.11, depending on the type of the iterated factorization. We recall some results that we use to prove these lemmas.

Lemma 3.5 (cf. [2, Lemma 8.1.4]). Let $S \in \mathrm{DS}$ and let $e \in E(S)$ and $u \in S$ such that $u \geq_{\mathcal{J}}$ e. Then euRe $\mathcal{L} u e$.

Corollary 3.6. Let $S \in \mathrm{DA}$ and let $e, f \in E(S)$ and $u \in S$ be such that $u \geq \mathcal{J}$ eJ $f$. Then euf $=e f$.

Corollary 3.7 (cf. [2, Theorem 8.1.7]). Let $S$ be a pro-DA semigroup and let $r, s, t \in S$ be such that $c(s) \subseteq c(r)=c(t)$. Then $r^{\omega} s t^{\omega}=r^{\omega} t^{\omega}$. 
Proposition 3.8 (cf. [2, Theorem 8.1.10]). Given $w \in \bar{\Omega}_{A} \mathrm{DA}$, then $w$ is idempotent if and only if $\left[\begin{array}{l}w \\ u\end{array}\right] \in\{0, \infty\}$, for any $u \in A^{+}$, where $\left[\begin{array}{l}w \\ u\end{array}\right]$ is the supremum of the integers $r$ such that $u^{r}$ is a subword of $w$.

We are now ready to prove the convergence of the infinite product in the iterated central basic factorization of type 1 of $w$, as stated in the following lemma:

Lemma 3.9. Given $w \in \bar{\Omega}_{A} \mathrm{DA} \backslash\{1\}$, if $w$ has an infinite iterated central basic factorization of type 1 , then $w=\vec{\prod}_{k \geq 1}\left(\alpha_{k} a_{k}\right) \cdot \overleftarrow{\prod}_{k \geq 1}\left(b_{k} \beta_{k}\right)$

Proof. The successive iterations of the central basic factorization of type 1 of $w$ are $w=\vec{\prod}_{k=1}^{n}\left(\alpha_{k} a_{k}\right) \cdot \gamma_{n} \cdot \overleftarrow{\prod}_{k=1}^{n}\left(b_{k} \beta_{k}\right)$, for all $n$. By compactness, there exists a subsequence $\left(\vec{\prod}_{k=1}^{m}\left(\alpha_{k} a_{k}\right), \gamma_{m}, \overleftarrow{\prod}_{k=1}^{m}\left(b_{k} \beta_{k}\right)\right)_{m \in M}$ that converges to some $(\alpha, \gamma, \beta)$. By Proposition 3.8, $\alpha$ and $\beta$ are idempotents, since $c\left(\alpha_{k} a_{k}\right)=c(\alpha)$ and $c\left(b_{k} \beta_{k}\right)=c(\beta)$, for all $k$, and also $\alpha \mathcal{J} \beta$, by [2, Theorem 8.1.7], since they have the same content. It follows, by Corollaries 3.6 and 3.4, that $w=\alpha \gamma \beta=\alpha \beta=\vec{\prod}_{k \geq 1}\left(\alpha_{k} a_{k}\right) \cdot \overleftarrow{\prod}_{k \geq 1}\left(b_{k} \beta_{k}\right)$ since $c(\gamma) \subseteq c(\alpha)=c(\beta)$.

To show that the infinite product in the iterated central basic factorization of type 2 of $w$ converges we need to beware of the fact that the content of the factors $\alpha_{k} a_{k}$ and $b_{k} \beta_{k}$ could decrease. In fact, the following lemma shows that the sequence of these contents stabilizes.

Lemma 3.10. Let $w \in \bar{\Omega}_{A} \mathrm{DA} \backslash\{1\}$ be such that the iterated central basic factorization of type 2 of $w$ is infinite, $\mathrm{I}_{2} \operatorname{CBF}(w)=\alpha_{1} a_{1} \alpha_{2} a_{2} \cdots \cdots b_{2} \beta_{2} b_{1} \beta_{1}$. Then there exists $N \in \mathbb{N}$ such that, if $i \geq N, c\left(\alpha_{i} a_{i}\right)$ and $c\left(b_{i} \beta_{i}\right)$ are constant and equal, for all $i$. Moreover, the pseudowords $w_{i}=\alpha_{i} a_{i} \alpha_{i+1} a_{i+1} \cdots \cdots b_{i+1} \beta_{i+1} b_{i} \beta_{i}$, with $i \geq N$, have a standard central basic factorization.

Proof. We consider the sequence $\left(w_{n}=\gamma_{n-1}=\alpha_{n} a_{n} \cdots \cdots b_{n} \beta_{n}\right)_{n \geq 1}$ of elements of $\bar{\Omega}_{A} \mathrm{DA} \backslash\{1\}$. We have $c\left(w_{1}\right)=c(w)$ and $c\left(w_{j}\right) \subseteq c\left(w_{i}\right)$, if $i<j$. Since $\mathrm{I}_{2} \mathrm{CBF}(w)$ is infinite and $A$ is a finite alphabet, it follows that, from a certain point on, the contents $c\left(w_{i}\right)$ must stabilize. Let $N$ be a integer such that, if $i, j \geq N$, then $c\left(w_{i}\right)=c\left(w_{j}\right)$. We recall that, if the central basic factorization of $w_{i}, \operatorname{CBF}\left(w_{i}\right)=$ $\alpha_{i} a_{i} \gamma_{i} b_{i} \beta_{i}$, is of the overlapped form, then $a_{i} \notin c\left(\gamma_{i} b_{i} \beta_{i}\right)$ and $b_{i} \notin c\left(\alpha_{i} a_{i} \gamma_{i}\right)$ and, therefore, $c\left(\gamma_{i}=w_{i+1}\right) \subsetneq c\left(w_{i}\right)$. On the other hand, if the central basic factorization of $w_{i}$ is degenerate, then $\mathrm{I}_{2} \operatorname{CBF}\left(w_{i}\right)$ is finite and, therefore, $\mathrm{I}_{2} \operatorname{CBF}(w)$ is also finite, which contradicts the hypothesis. So, if $i \geq N$, then $\operatorname{CBF}\left(w_{i}\right)$ is standard. By definition of standard central basic factorization and by the above, it follows that, if $i \geq N$, then $c\left(\alpha_{i} a_{i}\right)=c\left(b_{i} \beta_{i}\right)=c\left(w_{N}\right)$.

Finally, we note that, if $i<N$, then the central basic factorization of $w_{i}$ could be of the standard or of the overlapped form.

Lemma 3.11. Given $w \in \bar{\Omega}_{A} \mathrm{DA} \backslash\{1\}$, if $w$ has an infinite iterated central basic factorization of type 2 , then $w=\vec{\prod}_{k \geq 1}\left(\alpha_{k} a_{k}\right) \cdot \overleftarrow{\prod}_{k \geq 1}\left(b_{k} \beta_{k}\right)$.

Proof. Let $\mathrm{I}_{2} \operatorname{CBF}(w)=\alpha_{1} a_{1} \alpha_{2} a_{2} \cdots \cdots b_{2} \beta_{2} b_{1} \beta_{1}$, let $N$ be an integer satisfying the condition of Lemma 3.10 and let $w_{N}=\alpha_{N} a_{N} \alpha_{N+1} a_{N+1} \cdots \cdots b_{N+1} \beta_{N+1} b_{N} \beta_{N}$. Note that, by Lemma 3.10, $c\left(\alpha_{k} a_{k}\right)=c\left(w_{N}\right)=c\left(b_{k} \beta_{k}\right)$, for all $k \geq N$. So, the iterated central basic factorization of type 2 of $w_{N}$ coincides with the iterated central basic factorization of type 1 of $w_{N}$. Applying Lemma 3.9 to this iterated 
factorization, it follows that $w_{N}=\vec{\prod}_{k \geq N}\left(\alpha_{k} a_{k}\right) \cdot \overleftarrow{\prod}_{k \geq N}\left(b_{k} \beta_{k}\right)$ and, therefore, $w=$ $\vec{\prod}_{k \geq 1}\left(\alpha_{k} a_{k}\right) \cdot \overleftarrow{\prod}_{k \geq 1}\left(b_{k} \beta_{k}\right)$

Example 3.12. The iterated central basic factorizations of $w=a^{\omega} b^{\omega} c^{2}(a b)^{\omega} c a b c^{\omega}$ of type 1 and of type 2 are, respectively:

$$
\mathrm{I}_{1} \operatorname{CBF}(w)=a^{\omega} b^{\omega} \cdot \underline{c} \cdot c a \cdot \underline{b} \cdot(a b)^{\omega} \cdot \underline{a} \cdot b c \cdot \underline{a} \cdot b c^{\omega}
$$

and $\mathrm{I}_{2} \operatorname{CBF}(w)=a^{\omega} b^{\omega} \cdot \underline{c} \cdot c a \cdot \underline{b} \cdot a \cdot \underline{b} \cdot a \cdot \underline{b} \cdots \cdots \underline{a} \cdot b \cdot \underline{a} \cdot b \cdot \underline{a} \cdot b c \cdot \underline{a} \cdot b c^{\omega}$.

Note that $\mathrm{I}_{1} \mathrm{CBF}(w)$ is finite, standard and of length 2 while $\mathrm{I}_{2} \mathrm{CBF}(w)$ is infinite.

The next step in our factorizations consists in applying successively the corresponding factorization to each factor $\alpha_{i}, \beta_{i}$ and $\gamma_{n}$, the latter only in the case of a finite iterated central basic factorization of type 1. We observe, by Proposition 3.13, that this process is finite. For that purpose, we define two operations in $\bar{\Omega}_{A} \mathrm{DA}, o p^{1}$ and $o p^{2}$, as follows. We start with $o p^{1}$ :

(i) Let $u=a_{1} \cdots a_{n} b_{n} \cdots b_{1} \in A^{+}$and $\alpha_{1}, \ldots, \alpha_{n}, \gamma_{n}, \beta_{n}, \ldots, \beta_{1} \in \bar{\Omega}_{A}$ DA be such that, for each $i<n, a_{i} \notin c\left(\alpha_{i}\right), b_{i} \notin c\left(\beta_{i}\right), \alpha_{i} a_{i}$ and $b_{i} \beta_{i}$ have the same content as the product $\alpha_{1} a_{1} \cdots \alpha_{n} a_{n} \gamma_{n} b_{n} \beta_{n} \cdots b_{1} \beta_{1}$ and, for $i=n$, either $a_{n}, b_{n}, \alpha_{n} a_{n}, b_{n} \beta_{n}$ satisfy the same conditions, or $a_{n} \notin c\left(\gamma_{n}\right) \cup\left\{b_{n}\right\} \cup c\left(\beta_{n}\right)$, $b_{n} \notin c\left(\alpha_{n}\right) \cup\left\{a_{n}\right\} \cup c\left(\gamma_{n}\right)$, and $\alpha_{n} a_{n} \gamma_{n} b_{n}$ and $a_{n} \gamma_{n} b_{n} \beta_{n}$ have the same content as $\alpha_{1} a_{1} \cdots \alpha_{n} a_{n} \gamma_{n} b_{n} \beta_{n} \cdots b_{1} \beta_{1}$. We define $o p_{u}^{1}\left(\alpha_{1}, \ldots, \alpha_{n}, \gamma_{n}, \beta_{n}, \ldots, \beta_{1}\right)=$ $\alpha_{1} a_{1} \cdots \alpha_{n} a_{n} \gamma_{n} b_{n} \beta_{n} \cdots b_{1} \beta_{1}$.

(ii) Let $u=a_{1} \cdots a_{n} b_{n-1} \cdots b_{1} \in A^{+}$and $\alpha_{1}, \ldots, \alpha_{n}, \beta_{n}, \ldots, \beta_{1} \in \bar{\Omega}_{A} \mathrm{DA}$ be such that, for all $i, a_{i} \notin c\left(\alpha_{i}\right), b_{i} \notin c\left(\beta_{i}\right), a_{n} \notin c\left(\beta_{n}\right)$ and $\alpha_{i} a_{i}, b_{i} \beta_{i}$ and $a_{n} \beta_{n}$ have the same content as the product $\alpha_{1} a_{1} \cdots \alpha_{n} a_{n} \beta_{n} \cdots b_{1} \beta_{1}$. We define $o p_{u}^{1}\left(\alpha_{1}, \ldots, \alpha_{n}, \beta_{n}, \ldots, \beta_{1}\right)=\alpha_{1} a_{1} \cdots \alpha_{n} a_{n} \beta_{n} \cdots b_{1} \beta_{1}$.

(iii) Let $u=\vec{\prod}_{i \geq 1} a_{i} \cdot \overleftarrow{\prod}_{i \geq 1} b_{i} \in A^{\omega+\omega^{*}}$ and $\alpha_{1}, \alpha_{2}, \ldots, \beta_{1}, \beta_{2}, \ldots \in \bar{\Omega}_{A} \mathrm{DA}$ be such that, for all $i, a_{i} \notin c\left(\alpha_{i}\right), b_{i} \notin c\left(\beta_{i}\right)$ and $\alpha_{i} a_{i}$ and $b_{i} \beta_{i}$ have the same content as the product $\vec{\prod}_{k \geq 1}\left(\alpha_{k} a_{k}\right) \cdot \overleftarrow{\prod}_{k \geq 1}\left(b_{k} \beta_{k}\right)$. We define $o p_{u}^{1}\left(\alpha_{1}, \alpha_{2}, \ldots, \ldots, \beta_{2}, \beta_{1}\right)=\vec{\prod}_{i \geq 1}\left(\alpha_{i} a_{i}\right) \cdot \overleftarrow{\prod}_{i \geq 1}\left(b_{i} \beta_{i}\right)$.

Similarly, we define $o p^{2}$, but now assuming that the contents could decrease:

(i) Let $u=a_{1} \cdots a_{n} b_{n} \cdots b_{1} \in A^{+}$and $\alpha_{1}, \ldots, \alpha_{n}, \beta_{n}, \ldots, \beta_{1} \in \bar{\Omega}_{A}$ DA be such that, for each $i<n, a_{i} \notin c\left(\alpha_{i}\right), b_{i} \notin c\left(\beta_{i}\right), \alpha_{i} a_{i}$ and $b_{i} \beta_{i}$ have the same content as the product $\alpha_{i} a_{i} \cdots \alpha_{n} a_{n} b_{n} \beta_{n} \cdots b_{i} \beta_{i}$ and, for $i=n$, either $a_{n} \notin$ $c\left(\alpha_{n}\right), b_{n} \notin c\left(\beta_{n}\right)$, and $\alpha_{n} a_{n}$ and $b_{n} \beta_{n}$ have the same content as $\alpha_{n} a_{n} b_{n} \beta_{n}$, or $a_{n} \notin\left\{b_{n}\right\} \cup c\left(\beta_{n}\right), b_{n} \notin c\left(\alpha_{n}\right) \cup\left\{a_{n}\right\}$ and $\alpha_{n} a_{n} b_{n}$ and $a_{n} b_{n} \beta_{n}$ have the same content as $\alpha_{n} a_{n} b_{n} \beta_{n}$. We define $o p_{u}^{2}\left(\alpha_{1}, \ldots, \alpha_{n}, \beta_{n}, \ldots, \beta_{1}\right)=$ $\alpha_{1} a_{1} \cdots \alpha_{n} a_{n} b_{n} \beta_{n} \cdots b_{1} \beta_{1}$.

(ii) Let $u=a_{1} \cdots a_{n} b_{n-1} \cdots b_{1} \in A^{+}$and $\alpha_{1}, \ldots, \alpha_{n}, \beta_{n}, \ldots, \beta_{1} \in \bar{\Omega}_{A} \mathrm{DA}$ be such that, for all $i, a_{i} \notin c\left(\alpha_{i}\right), b_{i} \notin c\left(\beta_{i}\right), a_{n} \notin c\left(\beta_{n}\right)$, and $\alpha_{i} a_{i}$ and $b_{i} \beta_{i}$ have the same content as the product $\alpha_{i} a_{i} \cdots \alpha_{n} a_{n} \beta_{n} \cdots b_{i} \beta_{i}$. We define $o p_{u}^{2}\left(\alpha_{1}, \ldots, \alpha_{n}, \beta_{n}, \ldots, \beta_{1}\right)=\alpha_{1} a_{1} \cdots \alpha_{n} a_{n} \beta_{n} \cdots b_{1} \beta_{1}$.

(iii) Let $u=\vec{\prod}_{i \geq 1} a_{i} \cdot \overleftarrow{\prod}_{i \geq 1} b_{i} \in A^{\omega+\omega^{*}}$ and $\alpha_{1}, \alpha_{2}, \ldots, \beta_{1}, \beta_{2}, \ldots \in \bar{\Omega}_{A} \mathrm{DA}$ be such that, for all $i, a_{i} \notin c\left(\alpha_{i}\right), b_{i} \notin c\left(\beta_{i}\right)$ and $\alpha_{i} a_{i}$ and $b_{i} \beta_{i}$ have the same content as the product $\vec{\prod}_{k \geq i}\left(\alpha_{k} a_{k}\right) \cdot \overleftarrow{\prod}_{k \geq i}\left(b_{k} \beta_{k}\right)$. We define $o p_{u}^{2}\left(\alpha_{1}, \alpha_{2}, \ldots, \ldots, \beta_{2}, \beta_{1}\right)=\vec{\prod}_{i \geq 1}\left(\alpha_{i} a_{i}\right) \cdot \overleftarrow{\prod}_{i \geq 1}\left(b_{i} \beta_{i}\right)$. 
Proposition 3.13. Each element of $\bar{\Omega}_{A} \mathrm{DA}$ can be obtained from 1 by applying successively one of the described operations a number of times that does not exceed $|A|$.

Proof. Each $w \in \bar{\Omega}_{A} \mathrm{DA} \backslash\{1\}$ has an iterated central basic factorization that involves a finite (or infinite) product of letters $a_{i}$ and $b_{i}$, which depends on the type of the iterated factorization. As the content of each factor is strictly contained in $c(w)$, the result follows by induction on $|c(w)|$.

We end this section by presenting some characterizations of the implicit operations on DA connected with its iterated central basic factorizations.

Let $\| w \prod_{i}$ be the number of iterations until we obtain the iterated central basic factorization of type $i$ of $w$, with $i \in\{1,2\}$. Note that $\llbracket w \rrbracket_{i} \in \mathbb{N} \cup\{\infty\}$. We denote by $\|w\|_{i}$ the greatest integer $n$ such that $c\left(\alpha_{n} a_{n}\right)=c\left(b_{n} \beta_{n}\right)=c(w)$ and $\alpha_{n} a_{n}$ and $b_{n} \beta_{n}$ are disjoint, in the iterated central basic factorization of type $i$ of $w$. If this maximum does not exist, we set $\|w\|_{i}=\infty$. If this condition does not occur for any integer $n$, we set $\|w\|_{i}=0$. Note that $\|w\|_{1}=\|w\|_{1}$ for the standard and the overlapped case, and $\llbracket w \rrbracket_{1}-1=\|w\|_{1}$ in the degenerate case. Moreover, we have $\|w\|_{1}=\|w\|_{2}$. The following statements are formulated for the iterated central basic factorization of type 2 , although we have similar results for the iterated central basic factorization of type 1 . We leave the details to the reader. From hereon, we use the notation $\llbracket w \rrbracket$ and $\|w\|$ instead of $\llbracket w \prod_{2}$ and $\|w\|_{2}$, respectively.

Lemma 3.14. Let $u, v \in \bar{\Omega}_{A} \mathrm{DA} \backslash\{1\}$. If $c(u)=c(v)$, then $\|u v\| \geq\|u\|+\|v\|$.

Proof. Let $u=\alpha_{u_{1}} a_{u_{1}} \cdots \alpha_{u_{m}} a_{u_{m}} \gamma_{u_{m}} b_{u_{m}} \beta_{u_{m}} \cdots b_{u_{1}} \beta_{u_{1}}$ and $v=\alpha_{v_{1}} a_{v_{1}} \cdots \alpha_{v_{n}}$. $a_{v_{n}} \gamma_{v_{n}} b_{v_{n}} \beta_{v_{n}} \cdots b_{v_{1}} \beta_{v_{1}}$ be, respectively, the $m$-iteration and the $n$-iteration of the central basic factorization of $u$ and $v$, with $\|u\|=m$ and $\|v\|=n$, for $m, n \in \mathbb{N}$. Note that $c\left(\alpha_{u_{i}} a_{u_{i}}\right)=c\left(b_{u_{i}} \beta_{u_{i}}\right)=c(u)=c(v)=c\left(\alpha_{v_{j}} a_{v_{j}}\right)=c\left(b_{v_{j}} \beta_{v_{j}}\right)$ for all $i \leq m$ and $j \leq n$. Since $u v=\alpha_{u_{1}} a_{u_{1}} \cdots a_{u_{m}} \gamma_{u_{m}} b_{u_{m}} \cdots b_{u_{1}} \beta_{u_{1}} \cdot \alpha_{v_{1}} a_{v_{1}} \cdots a_{v_{n}} \gamma_{v_{n}} b_{v_{n}} \cdots b_{v_{1}} \beta_{v_{1}}$, it is easy to see that $\|u v\| \geq m+n=\|u\|+\|v\|$. The case where $\|u\|=\infty$ or $\|v\|=\infty$ is similar.

We define the cumulative content of $w \in \bar{\Omega}_{A} \mathrm{DA}$, and we denote it by $\vec{c}(w)$, to be the empty set, if $\mathrm{I}_{2} \operatorname{CBF}(w)$ is finite, or the set of all letters $a \in A$ such that there exists $N \in \mathbb{N}$ such that, for all $n \geq N, a \in c\left(\alpha_{n} a_{n}\right)=c\left(b_{n} \beta_{n}\right)$, in the case where $\mathrm{I}_{2} \operatorname{CBF}(w)$ is infinite.

Proposition 3.15. Let $w \in \bar{\Omega}_{A} \mathrm{DA} \backslash\{1\}$. The following conditions are equivalent:

(i) $\mathrm{DA} \models w^{2}=w$;

(ii) $\|w\|=\infty$;

(iii) $c(w)=\vec{c}(w)$.

Proof. $(i) \Rightarrow($ ii $)$ : Suppose that $w$ is idempotent. By Lemma 3.14, we have $\|w\|=$ $\left\|w^{2}\right\| \geq 2\|w\|$ which implies $\|w\|=\infty$ or $\|w\|=0$. Since $\left\|w^{2}\right\|>0$, for all $w \in \bar{\Omega}_{A} \mathrm{DA} \backslash\{1\}$, then we have $\|w\|=\infty$.

$(i i) \Rightarrow(i)$ : Suppose that $\|w\|=\infty$, i.e., $w=\vec{\prod}_{k \geq 1}\left(\alpha_{k} a_{k}\right) \cdot \overleftarrow{\prod}_{k \geq 1}\left(b_{k} \beta_{k}\right)$ with $c\left(\alpha_{k} a_{k}\right)=c\left(b_{k} \beta_{k}\right)=c(w)$, for all $k$. By Proposition 3.8, it follows that $w$ is an idempotent of $\bar{\Omega}_{A}$ DA. Let $\varphi: \bar{\Omega}_{A} \mathrm{DA} \rightarrow S$ be a continuous homomorphism in a finite monoid $S \in \mathrm{DA}$. Then $\varphi(w)$ is an idempotent. Thus DA $\models w^{2}=w$.

(ii) $\Leftrightarrow($ iii $)$ : It follows immediately from the definition of cumulative content of $w$. 
Lemma 3.16. Let $w=x y z \in \bar{\Omega}_{A} \mathrm{DA}$ with $c(x), c(z) \subsetneq c(w)$. Then $\|w\| \leq\|y\|+1$.

Proof. If $\|y\|=\infty$ or $x$ and $z$ are the empty word, the result is trivial. Otherwise, we proceed by induction on $(|c(y)|,\|y\|)$ where the pairs are in the lexicographic order. Suppose that $|c(y)|=1$. Since $c(x), c(z) \subsetneq c(w)$, there exists a letter in $c(y) \cup c(z)$ that does not belongs to $c(x)$ and there exists a letter in $c(x) \cup c(y)$ that does not belong to $c(z)$. By definition of central basic factorization, the following cases can occur:

(i) $\operatorname{CBF}(w)=(\alpha, a, \gamma, b, \beta)$ of the standard form: In this case, the letters $a$ and $b$ belong to the content of $y$ (otherwise, $c(x)=c(w)$ or $c(z)=c(w)$ ). Since $|c(y)|=1$, we have $a=b$. Moreover, $a \notin c(x)=c(z)$ and $c(\gamma)=$ $\{a\} \subsetneq c(w)$, because $\gamma$ is a factor of $y$ and $c(y)=\{a\}$. It follows that $\|w\|=$ 1.

(ii) $\operatorname{CBF}(w)=(\alpha, a, \gamma, b, \beta)$ of the overlapped form: It follows, immediately, that $\|w\|=0$.

(iii) $\operatorname{CBF}(w)=(\alpha, a, \beta)$ of the degenerate form: It follows, immediately, that $\|w\|=$ 0 .

Now, suppose that $|c(y)|>1$. Let $y_{1}$ be a prefix of $y z$ and let $y_{2}$ be a suffix of $x y$ such that $y_{1}$ is minimal for $c\left(x y_{1}\right)=c(w)$ and $y_{2}$ is minimal for $c\left(y_{2} z\right)=c(w)$. Note that $x y_{1}$ is one of the products $\alpha a$ or $\alpha a \gamma b$, according to the $\operatorname{CBF}(w)$ is of the standard or of the degenerate form, in the first case, or of the overlapped form, in the second case. We have the dual result for the factor $y_{2} z$ and, therefore, the existence of these factors is justified. We also note that $y_{1}$ and $y_{2}$ are non-empty words, by the hypothesis $c(x), c(z) \subsetneq c(w)$. If $y_{1}$ is not a prefix of $y$ or $y_{2}$ is not a suffix of $y$, then the central basic factorization of $w$ is overlapped and, therefore, $\|w\|=0$. Suppose that $y_{1}$ is a prefix of $y$ and $y_{2}$ is a suffix of $y$. Two cases can occur: $y=y_{1} y^{\prime} y_{2}$, with $y^{\prime}$ possibly empty, or $y_{1}$ and $y_{2}$ are not disjoint factors of $y$. In the latter case, the central basic factorization of $w$ is of the overlapped form or of the degenerate form and, therefore, $\|w\|=0$. Suppose that $y=y_{1} y^{\prime} y_{2}$, with $y^{\prime}$ possibly empty. If $c\left(y^{\prime}\right) \subsetneq c(w)$, then $\|w\|=1$ and the result follows. Suppose that $c\left(y^{\prime}\right)=c(w)$ and, therefore, $\|w\|=\left\|y^{\prime}\right\|+1$. We consider the central basic factorization of $y, \operatorname{CBF}(y)$. The cases where $\operatorname{CBF}(y)$ are of the degenerate or of the overlapped form are trivial, because $\left\|y^{\prime}\right\|=0$ (note that $c\left(y^{\prime}\right)=c(y)$ ). Suppose that $\operatorname{CBF}(y)=(\alpha, a, \gamma, b, \beta)$ is of the standard form. Then there exist $y_{1}^{\prime}$ and $y_{2}^{\prime}$ such that $\alpha a=y_{1} y_{1}^{\prime}, b \beta=y_{2}^{\prime} y_{2}$ and $y_{1}^{\prime} \gamma y_{2}^{\prime}=y^{\prime}$. We have $c(\gamma) \subsetneq c(y)$ or $\|\gamma\|=\|y\|-1$. If $c(\gamma) \subsetneq c(y)$, then $\|y\|=1,\left\|y^{\prime}\right\| \leq 1$ and $\|w\| \leq 2$. Otherwise, it follows that $c(\gamma)=c(y)=c\left(y^{\prime}\right)=c(w)$. If $c\left(y_{1}^{\prime}\right), c\left(y_{2}^{\prime}\right) \subsetneq c(\gamma)$, by induction hypothesis, it follows that $\left\|y^{\prime}\right\| \leq\|\gamma\|+1=\|y\|$. Thus $\|w\| \leq\|y\|+1$. Otherwise, $c\left(y_{1}^{\prime}\right)=c(\gamma), c\left(y_{2}^{\prime}\right)=c(\gamma)$, or both occur simultaneously. Suppose, by symmetry, that $c\left(y_{1}^{\prime}\right)=c(\gamma)=c(w)$. Since $\alpha a=y_{1} y_{1}^{\prime}$ is the minimum prefix of $y$ such that $c(\alpha a)=c(w)$, it follows that $y_{1}^{\prime}$ is the minimum prefix of $y^{\prime}$ such that $c\left(y_{1}^{\prime}\right)=c(w)$. It follows that $\|w\|=\left\|y^{\prime}\right\|+1 \leq\|\gamma\|+2=\|y\|+1$.

Corollary 3.17. Let $w=x_{1} \cdots x_{r} \in \bar{\Omega}_{A} \mathrm{DA}$ with $c\left(x_{i}\right) \subsetneq c(w)$, for all $i$. Then $\|w\|<\frac{r}{2}$.

Proof. If $r=2$, then $\|w\|=0$, since the central basic factorization of $w$ is of the overlapped form. If $r=3$, then, depending of the type of the central basic factorization of $w$, we have $\|w\|=1$ or $\|w\|=0$. If $r>3$, by the previous lemma, we have $\|w\| \leq\left\|x_{2} \cdots x_{r-1}\right\|+1$. It follows, by induction on $r$, that 
$\|w\| \leq \frac{r-3}{2}+1=\frac{r}{2}-\frac{1}{2}$, in the case where $r$ is odd, or $\|w\| \leq \frac{r}{2}-1$, in the case where $r$ is even. In any case, $\|w\|<\frac{r}{2}$.

\section{Representation of implicit operations on DA}

4.1. Two tree representations. We present two distinct representations of the implicit operations on DA by trees. The first one comes from ideas used by Almeida and Weil [7] for representing implicit operations over R. In the second one, we extend to DA some techniques developed by Almeida and Zeitoun [8] to solve the word problem for $\omega$-terms over R. We end the subsection by giving a more compact representation of the second tree representation using automata.

4.1.1. Representation by finite-height trees. Let $A$ be a finite alphabet. We define the set $T_{1}(A)$ to be a set of trees of finite height and with a number of vertices that may be infinite. The set $T_{1}(A)$ consists of all trees with a root and satisfying the following conditions:

(1) The vertices which are direct descendants of a vertex $v$, and that we call the progeny of $v$, are ordered with order type $\mathbf{n}$, with $n$ finite and odd, or with order type $\omega+\omega^{*}$.

(2) The direct descendants (or sons) of a vertex are ordered as follows: reading from left to right, and also from right to left, they switch between a vertex which we call node and a vertex which we call leaf and always starting, whether we read from the left or from the right, by a node.

(3) A node has one and only one of the following properties: either it has descendants, or it is labeled by 1 (in this case we call it a degenerate node).

(4) A leaf does not have descendants and it is labeled by a letter in $A$.

(5) The content of a leaf consists of its label; the content of a node is the set of labels of its descendants leaves; the content of a degenerate node is $\emptyset$.

For each non-degenerate node $v$, let $v_{i}$ and $f_{i}$ be, respectively, the $i$-th node and the $i$-th leaf, when we read from left to right, in the progeny of $v$ and let $v_{i}^{\prime}$ and $f_{i}^{\prime}$ be, respectively, the $i$-th node and the $i$-th leaf, when we read from right to left, in the progeny of $v$. Three distinct cases can occur, depending if the progeny of $v$ has order type $\omega+\omega^{*}, 4 \mathbf{m}-1$ or $4 \mathbf{m}+1$ :

(6) The case $\omega+\omega^{*}$ : For all $i<\omega$, we have $c\left(f_{i}\right) \notin c\left(v_{i}\right), c\left(f_{i}^{\prime}\right) \notin c\left(v_{i}^{\prime}\right)$ and $c\left(v_{i}\right) \cup c\left(f_{i}\right)=c\left(f_{i}^{\prime}\right) \cup c\left(v_{i}^{\prime}\right)=c(v)$.

(7) The case $4 \mathbf{m}-1$ : For all $i \leq m$, we have $c\left(f_{i}\right) \notin c\left(v_{i}\right), c\left(f_{i}^{\prime}\right) \notin c\left(v_{i}^{\prime}\right)$ and $c\left(v_{i}\right) \cup c\left(f_{i}\right)=c\left(f_{i}^{\prime}\right) \cup c\left(v_{i}^{\prime}\right)=c(v)$.

(8) The case $4 \mathbf{m}+1$ : For all $i<m$, we have $c\left(f_{i}\right) \notin c\left(v_{i}\right), c\left(f_{i}^{\prime}\right) \notin c\left(v_{i}^{\prime}\right)$ and $c\left(v_{i}\right) \cup c\left(f_{i}\right)=c\left(f_{i}^{\prime}\right) \cup c\left(v_{i}^{\prime}\right)=c(v)$. For $f=f_{m}$ or $f=f_{m}^{\prime}$, then one and only one of the following cases can occur:

(8-a) $c\left(f_{m}\right) \notin c\left(v_{m}\right), c\left(f_{m}^{\prime}\right) \notin c\left(v_{m}^{\prime}\right), c\left(v_{m}\right) \cup c\left(f_{m}\right)=c\left(f_{m}^{\prime}\right) \cup c\left(v_{m}^{\prime}\right)=c(v)$ and $c\left(v_{m+1}\right) \neq c(v)$.

(8-b) $c\left(f_{m}\right) \notin c\left(v_{m+1}\right) \cup c\left(f_{m}^{\prime}\right) \cup c\left(v_{m}^{\prime}\right), c\left(f_{m}^{\prime}\right) \notin c\left(v_{m}\right) \cup c\left(f_{m}\right) \cup c\left(v_{m+1}\right)$ and $c\left(v_{m}\right) \cup c\left(f_{m}\right) \cup c\left(v_{m+1}\right) \cup c\left(f_{m}^{\prime}\right)=c\left(f_{m}\right) \cup c\left(v_{m+1}\right) \cup c\left(f_{m}^{\prime}\right) \cup c\left(v_{m}^{\prime}\right)=c(v)$.

Note that the contents of the successive descendants nodes of a branch strictly decrease. It follows that the height of $t \in T_{1}(A)$ is at most $|A|$.

We define the mapping $\varrho: T_{1}(A) \rightarrow \bar{\Omega}_{A} \mathrm{DA}$ as follows. Let $t \in T_{1}(A)$. We obtain $\varrho(t)$ as the ordered reading, from left to right, of the labels of the leaves of $t$. Formally, we define $\varrho(t)$ by induction on the height of $t$. If $t$ has height 0 , 
then $t$ is only a vertex, which is a degenerate node, and we set $\varrho(t)=1$. Suppose that $t$ has non-zero height. Let $a_{1}, \ldots, a_{n}, b_{n}, \ldots, b_{1}$ be the labels of the leaves that are direct descendants of the root (in case the number of leaves is finite and odd, we have $a_{1}, \ldots, a_{n}, b_{n-1}, \ldots, b_{1}$, and, in case that this number is infinite, we have $a_{1}, a_{2}, \ldots \ldots, b_{2}, b_{1}$, respectively) and let $t_{1}, \ldots, t_{n}, t_{n+1}, t_{n}^{\prime}, \ldots, t_{1}^{\prime}$ (respectively, $t_{1}, \ldots, t_{n}, t_{n}^{\prime}, \ldots, t_{1}^{\prime}$ and $\left.t_{1}, t_{2}, \ldots \ldots, t_{2}^{\prime}, t_{1}^{\prime}\right)$ be the subtrees attached to each direct descendant node of the root. Note that each subtree has height strictly lower than the height of $t$. Let

$$
\varrho(t)=\vec{\prod}_{i \geq 1}^{n}\left(\varrho\left(t_{i}\right) a_{i}\right) \cdot \varrho\left(t_{n+1}\right) \cdot \overleftarrow{\prod}_{i \geq 1}^{n}\left(b_{i} \varrho\left(t_{i}^{\prime}\right)\right)
$$

respectively

or

$$
\varrho(t)=\vec{\prod}_{i \geq 1}^{n}\left(\varrho\left(t_{i}\right) a_{i}\right) \cdot \varrho\left(t_{n}^{\prime}\right) \cdot \overleftarrow{\prod}_{i \geq 1}^{n-1}\left(b_{i} \varrho\left(t_{i}^{\prime}\right)\right)
$$

$$
\varrho(t)=\vec{\prod}_{i \geq 1}\left(\varrho\left(t_{i}\right) a_{i}\right) \cdot \overleftarrow{\prod}_{i \geq 1}\left(b_{i} \varrho\left(t_{i}^{\prime}\right)\right)
$$

Lemma 4.1. The factorization used in the definition of $\varrho(t)$ is the iterated central basic factorization of type 1 defined in the previous section.

Proof. It suffices to note that, by properties (6), (7) and (8) from the definition of tree $t \in T_{1}(A), a_{1}$ and $b_{1}$ are, respectively, the first occurrence of the last appearing letter when we read from left to right and from right to left. The other labels $a_{2}, \ldots, b_{2}$ in the progeny of the root satisfy the same condition in the subtree of $t$ which results from eliminating the first two and the last two branches that leave from the root and so on, until the content of the subtree decreases, as in the iteration of type 1 of the central basic factorization defined previously. Since, by Proposition 3.1 this factorization is unique, it follows that the factorization of $\varrho(t)$ is the iterated central basic factorization of type 1 .

Theorem 4.2. The mapping $\varrho: T_{1}(A) \rightarrow \bar{\Omega}_{A} \mathrm{DA}$ is a bijection.

Proof. Let $t$ and $\bar{t}$ be distinct elements in $T_{1}(A)$. Let $a_{1}, a_{2}, \ldots, b_{2}, b_{1}$ be the labels of the leaves which are direct descendants of the root of $t$ and let $t_{1}, t_{2}, \ldots, t_{2}^{\prime}$, $t_{1}^{\prime}$ be the subtrees attached to each direct descendant node of the root of $t$ (as we have defined previously). Similarly, we define $\bar{a}_{1}, \bar{a}_{2}, \ldots, \bar{b}_{2}, \bar{b}_{1}$ and $\bar{t}_{1}, \bar{t}_{2}, \ldots, \bar{t}_{2}^{\prime}, \bar{t}_{1}^{\prime}$ with respect to $\bar{t}$. Let $k$ be minimum with $t_{k} \neq \bar{t}_{k}, a_{k} \neq \bar{a}_{k}, t_{k}^{\prime} \neq \bar{t}_{k}^{\prime}$ or $b_{k} \neq \bar{b}_{k}$ and consider the $k$-th iteration of the central basic factorization of $\varrho(t)$ and $\varrho(\bar{t})$ :

$$
\varrho(t)=\varrho\left(t_{1}\right) a_{1} \cdots \varrho\left(t_{k}\right) a_{k} \gamma_{k} b_{k} \varrho\left(t_{k}^{\prime}\right) \cdots b_{1} \varrho\left(t_{1}^{\prime}\right)
$$

and

$$
\varrho(\bar{t})=\varrho\left(\bar{t}_{1}\right) \bar{a}_{1} \cdots \varrho\left(\bar{t}_{k}\right) \bar{a}_{k} \bar{\gamma}_{k} \bar{b}_{k} \varrho\left(\bar{t}_{k}^{\prime}\right) \cdots \bar{b}_{1} \varrho\left(\bar{t}_{1}^{\prime}\right) .
$$

Note that, if we have $2 n+1$ nodes in the progeny of the root, with $k=n+1$, i.e., when $t$ and $\bar{t}$ differ in the subtree attached at the node in the central position, we only iterate $k-1$ times. Proceeding by induction on the height of $t$, it follows that $\varrho\left(t_{k}\right) \neq \varrho\left(\bar{t}_{k}\right), a_{k} \neq \bar{a}_{k}, \varrho\left(t_{k}^{\prime}\right) \neq \varrho\left(\bar{t}_{k}^{\prime}\right)$ or $b_{k} \neq \bar{b}_{k}$. Since, by Proposition 3.1, the central basic factorization is unique, we deduce that $\varrho(t) \neq \varrho(\bar{t})$.

Let $w \in \bar{\Omega}_{A}$ DA. To verify that the mapping is onto, we proceed by induction on $|c(w)|$. If $|c(w)|=0$, then $w=1$ and $w=\varrho(t)$, where $t$ is a tree with just one vertex. If $|c(w)| \neq 0$, then $w=o p_{u}^{1}\left(\alpha_{1}, \alpha_{2}, \ldots, \beta_{2}, \beta_{1}\right)$, where $u=a_{1} a_{2} \cdots b_{2} b_{1}$ is an element in $A^{+} \cup A^{\omega+\omega^{*}}$ and $\alpha_{1}, \alpha_{2}, \ldots, \beta_{2}, \beta_{1}$ satisfy the conditions used to define 
$o p_{u}^{1}$ : for all $i, c(w)=c\left(\alpha_{i} a_{i}\right), c(w)=c\left(b_{i} \beta_{i}\right), a_{i} \notin c\left(\alpha_{i}\right)$ and $b_{i} \notin c\left(\beta_{i}\right)$. Moreover, if $|u|$ is finite and odd, then, since $a_{n}$ is the letter at the central position, we also have $a_{n} \notin c\left(\beta_{n}\right)$. If $|u|=n$ is finite and even and the central basic factorization of $w$ is of the overlapped form, we have that, for $k=n, a_{n} \notin c\left(\gamma_{n}\right) \cup\left\{b_{n}\right\} \cup c\left(\beta_{n}\right)$ and $b_{n} \notin c\left(\alpha_{n}\right) \cup\left\{a_{n}\right\} \cup c\left(\gamma_{n}\right)$. It follows that $c\left(\alpha_{i}\right)$ and $c\left(\beta_{i}\right)$ are strictly contained in $c(w)$, for all $i$. For all $i$, let $t_{i}$ and $t_{i}^{\prime}$ be elements in $T_{1}(A)$ such that $\varrho\left(t_{i}\right)=\alpha_{i}$ and $\varrho\left(t_{i}^{\prime}\right)=\beta_{i}$. Then $w=\varrho(t)$, where $t$ is the subtree of $T_{1}(A)$ whose leaves of the progeny of the root are labeled with $a_{1}, a_{2}, \ldots, b_{2}, b_{1}$ and whose nodes of this progeny have the subtrees $t_{1}, t_{2}, \ldots, t_{2}^{\prime}, t_{1}^{\prime}$ attached, respectively.

Example 4.3. The tree in $T_{1}(A)$ which represents $w=a^{\omega} b^{\omega} c^{2}(a b)^{\omega} c a b c^{\omega} \in \bar{\Omega}_{A} \mathrm{DA}$ is the following:

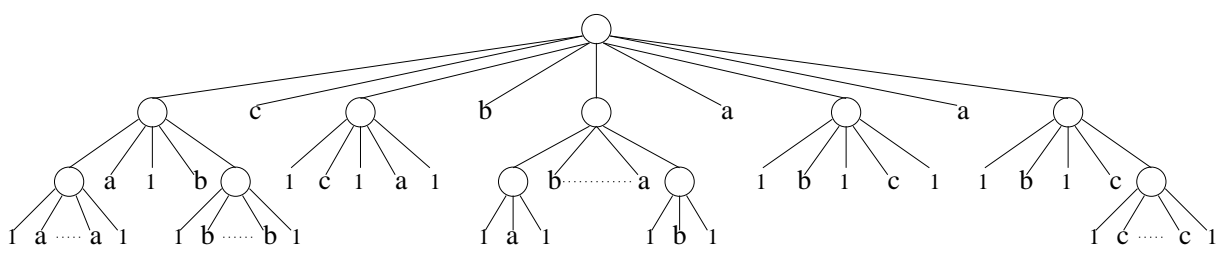

4.1.2. Representation by quasi-ternary trees. Let $A$ be a finite alphabet. We define a set $T_{2}(A)$ of quasi-ternary trees with labeled vertices, of which there may be infinitely many, and whose heights of the central branches can be infinite too. The set $T_{2}(A)$ consists of all trees with a root such that:

(i) The vertices are labeled with a pair of letters in $A$, or with a letter in $A$ (degenerate vertex), or with a letter $\varepsilon \notin A$ (final vertex).

(ii) The vertices which are labeled with a pair of letters have three direct descendants. The degenerate vertices have only two direct descendants. The final vertices do not have descendants.

We define recursively the content of a vertex $v, c(v)$, to be the union of the contents of their sons with the set of its labels. The left content of a vertex $v, c_{l}(v)$, is the content of its left son. Dually, one may define the right content of a vertex $v$, $c_{r}(v)$. In the case where the vertex is non-degenerate, we also define the central content of a vertex $v, c_{c}(v)$, to be the content of its central son.

The set $T_{2}(A)$ also satisfies the following condition:

(iii) For each non-final vertex $v$ one of the following cases occurs:

(iii-a) Non-degenerate case: Let $\left(m_{1}, m_{2}\right)$ be the label of $v$. Then only one of the following cases occurs: either

$$
\begin{aligned}
& m_{1} \notin c_{l}(v), m_{2} \notin c_{r}(v) \text { and } c_{l}(v) \cup\left\{m_{1}\right\}=\left\{m_{2}\right\} \cup c_{r}(v)=c(v), \\
& \quad \text { or } \\
& m_{1} \notin c_{c}(v) \cup\left\{m_{2}\right\} \cup c_{r}(v), m_{2} \notin c_{l}(v) \cup\left\{m_{1}\right\} \cup c_{c}(v) \text { and } \\
& c_{l}(v) \cup\left\{m_{1}\right\} \cup c_{c}(v) \cup\left\{m_{2}\right\}=\left\{m_{1}\right\} \cup c_{c}(v) \cup\left\{m_{2}\right\} \cup c_{r}(v)=c(v) .
\end{aligned}
$$

(iii-b) Degenerate case: Let $m$ be the label of $v$. We have $m \notin c_{l}(v), m \notin$ $c_{r}(v)$ and $c_{l}(v) \cup\{m\}=\{m\} \cup c_{r}(v)=c(v)$.

Observe that the contents of the vertices of the successive descendants from the right or from the left branches strictly decrease. We define the depth of $t \in T_{2}(A)$, $\mathfrak{d}(t)$, to be the maximum distance to the root of vertices which do not have as an 
ancestor a vertex from a central branch. It follows that the depth of $t$ is at most $|A|$, but it can be strictly smaller. As an example, $\mathfrak{d}\left(a_{1} \cdots a_{n}\right)=1$, with $a_{i} \neq a_{j}$ if $i \neq j$.

We define the mapping $\rho: T_{2}(A) \rightarrow \bar{\Omega}_{A}$ DA recursively as follows. Let $t \in T_{2}(A)$. If $t$ has non-zero depth, i.e., $t$ is just a vertex with label $\varepsilon$, we set $\rho(t)=1$. Otherwise, let $v_{0}, v_{1}$ and $v_{2}\left(v_{0}\right.$ and $v_{2}$ in the degenerate case) be the vertices descending directly from the root $v_{\varepsilon}$. For each vertex $v_{i}$, with $i \in\{0,1,2\}^{*}$, let $v_{i 0}$, $v_{i 1}$ and $v_{i 2}\left(v_{i 0}\right.$ and $v_{i 2}$ in the degenerate case) be the sons of $v_{i}$. Let $l_{(i, 0)}$ and $l_{(i, 2)}$ be the labels of $v_{i}$ (in the degenerate case, let $l_{(i, 0)}$ be this label). We denote by $t_{i 0}$, $t_{i 1}$ and $t_{i 2}$ the subtrees that begin at the sons $v_{i 0}, v_{i 1}$ and $v_{i 2}$, respectively. Then, $\rho(t)$ is described as follows:

$$
\rho(t)=\rho\left(t_{0}\right) \cdot l_{(\varepsilon, 0)} \cdot \rho\left(t_{10}\right) \cdot l_{(1,0)} \cdots \cdots l_{(1,2)} \cdot \rho\left(t_{12}\right) \cdot l_{(\varepsilon, 2)} \cdot \rho\left(t_{2}\right)
$$

if the central body of the tree is infinite. If it is finite, we have one of the following cases:

$$
\rho(t)=\rho\left(t_{0}\right) \cdot l_{(\varepsilon, 0)} \cdots \rho\left(t_{1^{n-1} 0}\right) \cdot l_{\left(1^{n-1}, 0\right)} \cdot l_{\left(1^{n-1}, 2\right)} \cdot \rho\left(t_{1^{n-1} 2}\right) \cdots l_{(\varepsilon, 2)} \cdot \rho\left(t_{2}\right)
$$

or

$$
\rho(t)=\rho\left(t_{0}\right) \cdot l_{(\varepsilon, 0)} \cdots \rho\left(t_{1^{n-1} 0}\right) \cdot l_{\left(1^{n-1}, 0\right)} \cdot \rho\left(t_{1^{n-1} 2}\right) \cdots l_{(\varepsilon, 2)} \cdot \rho\left(t_{2}\right) .
$$

Lemma 4.4. The factorization used in the definition of $\rho(t)$ is the iterated central basic factorization of type 2 defined above.

Proof. By Property (iii) from the tree's definition, we know that $l_{(\varepsilon, 0)}$ and $l_{(\varepsilon, 2)}$ are, respectively, the first occurrence of the last appearing letter when we read, respectively, from left to right and from right to left. The other labels from this factorization arise from the inductive process of applying the same factorization to the subtree of $t$ that has as a root the central son of the root of $t$. This process ends when the root of the subtree that we are considering is degenerate or, if it is not degenerate, it has as a central son a final vertex. By Proposition 3.1, this factorization is unique and the iteration ends with the same condition of the iterated central basic factorization of type 2 of $\rho(t)$. Thus the factorizations are equal.

Theorem 4.5. The mapping $\rho: T_{2}(A) \rightarrow \bar{\Omega}_{A} \mathrm{DA}$ is a bijection.

Proof. Let $t$ and $\bar{t}$ be distinct elements of $T_{2}(A)$. Let $k$ be minimum for $l_{\left(1^{k}, 0\right)} \neq$ $\bar{l}_{\left(1^{k}, 0\right)}, l_{\left(1^{k}, 2\right)} \neq \bar{l}_{\left(1^{k}, 2\right)}, t_{1^{k} 0} \neq \bar{t}_{1^{k} 0}$ or $t_{1^{k} 2} \neq \bar{t}_{1^{k} 2}$. Consider the $k+1$-iteration of the central basic factorization of $\rho(t)$ and $\rho(\bar{t})$ :

$$
\rho(t)=\rho\left(t_{0}\right) \cdot l_{(\varepsilon, 0)} \cdots \rho\left(t_{1^{k} 0}\right) \cdot l_{\left(1^{k}, 0\right)} \cdot \rho\left(t_{1^{k+1}}\right) \cdot l_{\left(1^{k}, 2\right)} \cdot \rho\left(t_{1^{k} 2}\right) \cdots l_{(\varepsilon, 2)} \cdot \rho\left(t_{2}\right)
$$

and

$$
\rho(\bar{t})=\rho\left(\bar{t}_{0}\right) \cdot \bar{l}_{(\varepsilon, 0)} \cdots \rho\left(\bar{t}_{1^{k} 0}\right) \cdot \bar{l}_{\left(1^{k}, 0\right)} \cdot \rho\left(\bar{t}_{1^{k+1}}\right) \cdot \bar{l}_{\left(1^{k}, 2\right)} \cdot \rho\left(\bar{t}_{1^{k} 2}\right) \cdots \bar{l}_{(\varepsilon, 2)} \cdot \rho\left(\bar{t}_{2}\right) .
$$

Proceeding by induction on the depth of $t$ and $\bar{t}$, it follows that $l_{\left(1^{k}, 0\right)} \neq \bar{l}_{\left(1^{k}, 0\right)}$, $l_{\left(1^{k}, 2\right)} \neq \bar{l}_{\left(1^{k}, 2\right)}, \rho\left(t_{1^{k} 0}\right) \neq \rho\left(\bar{t}_{1^{k} 0}\right)$ or $\rho\left(t_{1^{k} 2}\right) \neq \rho\left(\bar{t}_{1^{k} 2}\right)$. Since, by Proposition 3.1, this factorization is unique, it follows that $\rho(t) \neq \rho(\bar{t})$.

Let $w \in \bar{\Omega}_{A}$ DA. To verify that $\rho$ is onto, we proceed by induction on $|c(w)|$. If $|c(w)|=0$, then $w=1$ and $w=\rho(t)$, where $t$ is the tree with just one vertex labeled by $\varepsilon$. If $|c(w)| \neq 0$, then we consider the iterated central basic factorization of $w, w=\alpha_{1} a_{1} \alpha_{2} a_{2} \cdots \cdots b_{2} \beta_{2} b_{1} \beta_{1}$ (or one of the other previously described cases). Note that $c\left(\alpha_{k}\right)$ and $c\left(\beta_{k}\right)$ are strictly contained in $c(w)$, for all $k$. For all $k$, let $t_{1^{k-1} 0}$ and $t_{1^{k-1} 2}$ be elements in $T_{2}(A)$ such that $\rho\left(t_{1^{k-1} 0}\right)=\alpha_{k}$ and 
$\rho\left(t_{1^{k-1} 2}\right)=\beta_{k}$. Then $w=\rho(t)$, where $t$ is the tree in $T_{2}(A)$ whose central vertices are labeled with $\left(a_{1}, b_{1}\right),\left(a_{2}, b_{2}\right)$, etc., and with the subtrees $t_{1^{k-1} 0}$ and $t_{1^{k-1} 2}$, for each $k$, respectively attached.

Example 4.6. We have the following representation by a tree in $T_{2}(A)$ of the pseudoword $w=a^{\omega} b^{\omega} c^{2}(a b)^{\omega} c a b c^{\omega} \in \bar{\Omega}_{A}$ DA:

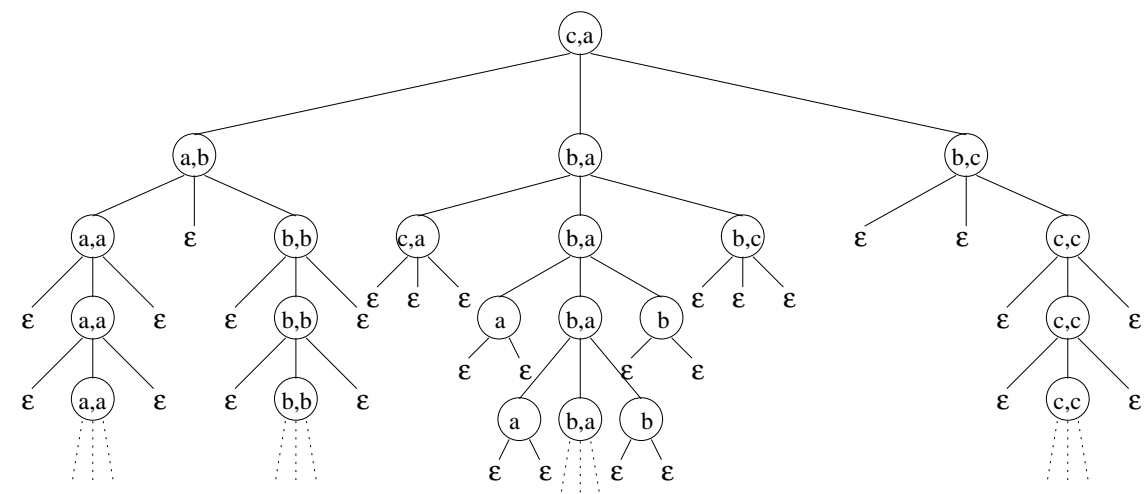

4.1.3. Representation by automata. It is sometimes convenient to compress the tree representation described in 4.1.2. We do it by identifying vertices which have the same attached subtrees. We begin with the definition of DA-automaton.

An $A$-labeled DA-automaton is a tuple $\mathcal{A}=(V, \rightarrow, q, F, \lambda)$ where $(V, \rightarrow, q, F)$ is a non-empty deterministic automaton (which may be infinite) over the alphabet $\mathbb{B}=\{0,1,2\}$ and $\lambda: V \rightarrow A \times A \cup A \cup\{\varepsilon\}$ is a total function. It also satisfies the following conditions:

(A1) The set of final states is $F=\lambda^{-1}(\varepsilon)$.

(A2) There are no outgoing transitions from $F$.

(A3) Let $v \in V \backslash F$. Then both $v .0$ and $v .2$ are defined. We also have $v .1$ defined if and only if $\lambda(v) \in A \times A$. Otherwise, $\lambda(v) \in A$.

(A4) Given $v \in V \backslash F$ with $\lambda(v)=\left(\lambda^{1}(v), \lambda^{2}(v)\right) \in A \times A$ we have one and only one of the following cases:

(i) $\lambda^{1}\left(v \cdot \mathbb{B}^{*}\right) \cup \lambda^{2}\left(v \cdot \mathbb{B}^{*}\right)=\left[\lambda^{1}\left(v \cdot 0 \mathbb{B}^{*}\right) \cup \lambda^{2}\left(v \cdot 0 \mathbb{B}^{*}\right)\right] \cup \lambda^{1}(v)$ and $\lambda^{1}\left(v \cdot \mathbb{B}^{*}\right) \cup \lambda^{2}\left(v \cdot \mathbb{B}^{*}\right)=\left[\lambda^{1}\left(v \cdot 2 \mathbb{B}^{*}\right) \cup \lambda^{2}\left(v \cdot 2 \mathbb{B}^{*}\right)\right] \cup{ }^{\circ} \lambda^{2}(v)$,

(ii) $\lambda^{1}\left(v \cdot \mathbb{B}^{*}\right) \cup \lambda^{2}\left(v \cdot \mathbb{B}^{*}\right)=\lambda^{1}(v) \stackrel{\circ}{\cup}\left[\lambda^{1}\left(v \cdot 1 \mathbb{B}^{*}\right) \cup \lambda^{2}\left(v \cdot 1 \mathbb{B}^{*}\right) \cup \lambda^{2}(v) \cup \lambda^{1}\left(v \cdot 2 \mathbb{B}^{*}\right) \cup\right.$ $\left.\lambda^{2}\left(v .2 \mathbb{B}^{*}\right)\right]$

and $\lambda^{1}\left(v \cdot \mathbb{B}^{*}\right) \cup \lambda^{2}\left(v \cdot \mathbb{B}^{*}\right)=\left[\lambda^{1}\left(v \cdot 0 \mathbb{B}^{*}\right) \cup \lambda^{2}\left(v \cdot 0 \mathbb{B}^{*}\right) \cup \lambda^{1}(v) \cup \lambda^{1}\left(v \cdot 1 \mathbb{B}^{*}\right) \cup\right.$ $\left.\lambda^{2}\left(v .1 \mathbb{B}^{*}\right)\right] \stackrel{\circ}{\cup} \lambda^{2}(v)$,

where we consider, for each vertex $v^{\prime}$ such that $\lambda\left(v^{\prime}\right) \in A, \lambda\left(v^{\prime}\right)=\lambda^{1}\left(v^{\prime}\right)$.

(A5) Given $v \in V \backslash F$ with $\lambda(v)=\lambda^{1}(v) \in A$ we have

$$
\begin{gathered}
\lambda^{1}\left(v \cdot \mathbb{B}^{*}\right) \cup \lambda^{2}\left(v \cdot \mathbb{B}^{*}\right)=\left[\lambda^{1}\left(v \cdot 0 \mathbb{B}^{*}\right) \cup \lambda^{2}\left(v \cdot 0 \mathbb{B}^{*}\right)\right] \stackrel{\circ}{\cup} \lambda^{1}(v) \\
\text { and } \lambda^{1}\left(v \cdot \mathbb{B}^{*}\right) \cup \lambda^{2}\left(v \cdot \mathbb{B}^{*}\right)=\left[\lambda^{1}\left(v \cdot 2 \mathbb{B}^{*}\right) \cup \lambda^{2}\left(v \cdot 2 \mathbb{B}^{*}\right)\right] \stackrel{\cup}{\cup} \lambda^{1}(v)
\end{gathered}
$$

where, again, we consider, for each vertex $v^{\prime}$ such that $\lambda\left(v^{\prime}\right) \in A, \lambda\left(v^{\prime}\right)=$ $\lambda^{1}\left(v^{\prime}\right)$.

The trees defined in 4.1.2 are DA-automata such that every state is reached from the initial state by a unique path. We call these trees DA-trees. 
Given a DA-automaton $\mathcal{A}=(V, \rightarrow, q, F, \lambda)$ and $v \in V$, the subautomaton of $\mathcal{A}$ with initial state $v$ is the DA-automaton $\mathcal{A}_{v}=\left(V \cap v \cdot \mathbb{B}^{*}, \rightarrow, v, F \cap v \cdot \mathbb{B}^{*}, \lambda_{\mid V \cap v \cdot \mathbb{B}^{*}}\right)$.

Observe that, by conditions (A4) and (A5), if $v$. $\alpha$ is defined, then $|\alpha|_{0}+|\alpha|_{2} \leq$ $|A|$. Moreover, each time we follow a transition labeled 0 or 2 , we end up in a subautomaton labeled by an alphabet strictly contained in the previous one. It follows that, if $p_{0} \stackrel{a_{1}}{\longrightarrow} p_{1} \stackrel{a_{2}}{\longrightarrow} \cdots \stackrel{a_{n}}{\longrightarrow} p_{n}=p_{0}$ is a closed path of $\mathcal{A}$, then $a_{i}=1$ for all $i=1, \ldots, n$.

We say that two DA-automata $\mathcal{A}=\left(V_{i}, \rightarrow_{i}, q_{i}, F_{i}, \lambda_{i}\right)(i=0,1)$ are isomorphic if there is a bijection $\varphi: V_{0} \rightarrow V_{1}$ such that, for all $v \in V$ and for all $a \in \mathbb{B}$, $\varphi(v \cdot a)=\varphi(v) . a$ and $\lambda_{1}(\varphi(v))=\lambda_{0}(v)$. if

We say that two DA-automata $\mathcal{A}=\left(V_{i}, \rightarrow_{i}, q_{i}, F_{i}, \lambda_{i}\right)(i=0,1)$ are $k$-equivalent

$$
\text { for all } \alpha \in \mathbb{B}^{*},|\alpha| \leq k \Longrightarrow \lambda_{0}\left(q_{0} \cdot \alpha\right)=\lambda_{1}\left(q_{1} \cdot \alpha\right) \text {. }
$$

We say that two DA-automata are equivalent if they are $k$-equivalent for all $k \geq 0$. We write $\mathcal{A}_{0} \sim_{k} \mathcal{A}_{1}$ to denote the $k$-equivalence and we set $\sim=\bigcap \sim_{k}$. Note that equivalent DA-trees are isomorphic, since, as we said above, each state is completely determined by the unique path starting at the initial state and ending at this state.

Lemma 4.7. Any DA-automaton has a unique (up to isomorphism of DA-trees) equivalent DA-tree.

Proof. Let $\mathcal{A}=(V, \rightarrow, q, F, \lambda)$ be a DA-automaton. We define the DA-tree $\mathcal{T}=$ $(W, \rightarrow, p, G, \nu)$ as follows. Let $W=\left\{\alpha \in \mathbb{B}^{*} \mid q . \alpha\right.$ is defined $\}, p=\varepsilon, \nu(\alpha)=\lambda(q . \alpha)$ and $G=\nu^{-1}(\varepsilon)$. If $q . \alpha 0$ and $q . \alpha 2$ are defined, i.e., if $\lambda(q . \alpha) \neq \varepsilon$, then we define the transitions $\alpha \stackrel{0}{\rightarrow} \alpha 0$ and $\alpha \stackrel{2}{\rightarrow} \alpha 2$. Moreover, if $q . \alpha 1$ is also defined, which corresponds to $\lambda(q . \alpha) \in A \times A$, then we have also the transition $\alpha \stackrel{1}{\rightarrow} \alpha 1$ of $\mathcal{T}$. It is easy to see that the properties (A1)-(A5) remain valid and the uniqueness of this construction results from the fact that equivalent DA-trees are isomorphic.

We call the unfolding of $\mathcal{A}$ the unique (up to isomorphism) DA-tree $\overrightarrow{\mathcal{A}}$ equivalent to the DA-automaton $\mathcal{A}$.

Corollary 4.8. Let $\mathcal{A}$ and $\mathcal{A}^{\prime}$ be DA-automata. Then $\mathcal{A} \sim \mathcal{A}^{\prime}$ if and only if $\overrightarrow{\mathcal{A}}=\overrightarrow{\mathcal{A}^{\prime}}$.

We define the value $\pi(\mathcal{A}) \in \bar{\Omega}_{A}$ DA of a DA-automaton $\mathcal{A}$ by $\pi(\mathcal{A})=\rho(\overrightarrow{\mathcal{A}})$. Given a DA-automaton $\mathcal{A}=(V, \rightarrow, q, F, \lambda)$ and $v \in V$, let $[v]=\pi\left(\mathcal{A}_{v}\right)$.

Lemma 4.9. Let $\mathcal{A}=(V, \rightarrow, q, F, \lambda)$ be a DA-automaton and $v \in V \backslash F$. Then, the central basic factorization of $[v]$ is $[v .0] \cdot \lambda^{1}(v) \cdot[v .1] \cdot \lambda^{2}(v) \cdot[v .2]$, if $v .1$ is defined, or $[v .0] \cdot \lambda^{1}(v) \cdot[v .2]$, otherwise. Thus, by uniqueness of the central basic factorization, we have, for $u, v \in V \backslash F$,

$$
[u]=[v] \Longrightarrow\left\{\begin{array}{l}
\lambda^{1}(u)=\lambda^{1}(v) \\
\lambda^{2}(u)=\lambda^{2}(v) \\
{[u .0]=[v .0]} \\
{[u .1]=[v .1]} \\
{[u .2]=[v .2]}
\end{array}\right.
$$

respectively,

$$
[u]=[v] \Longrightarrow\left\{\begin{array}{l}
\lambda^{1}(u)=\lambda^{1}(v) \\
{[u .0]=[v .0]} \\
{[u .2]=[v .2] .}
\end{array}\right.
$$


Proof. It suffices to proceed by induction on $c([v])$ taking into account the definition of central basic factorization.

We call the wrapping of a DA-automaton $\mathcal{A}=(V, \rightarrow, q, F, \lambda)$ the DA-automaton $[\mathcal{A}]=([V], \rightarrow,[q],[F], \nu)$ where:

(i) $[V]=\{[v] \mid v \in V\} \subseteq \bar{\Omega}_{A} \mathrm{DA}$;

(ii) $[v] .0=[v .0],[v] .1=[v .1]$ and $[v] .2=[v .2]$;

(iii) $\nu([v])=\lambda(v)$.

This automaton is obtained from $\mathcal{A}$ by identifying states representing the same pseudoword. For $w \in \bar{\Omega}_{A}$ DA we define the wrapped DA-automaton of $w$ to be $\mathcal{A}(w)=\left[\rho^{-1}(w)\right]$.

The value of a path $p_{0} \stackrel{\delta_{0}}{\longrightarrow} p_{1} \stackrel{\delta_{1}}{\longrightarrow} \cdots \stackrel{\delta_{n}}{\longrightarrow} p_{n+1}$ in a DA-automaton is the product $\prod_{i=0}^{n}\left(\delta_{i}, \lambda\left(p_{i}\right)\right) \in(\mathbb{B} \times(A \times A \cup A))^{*}$. The language $\mathcal{L}(v) \subseteq(\mathbb{B} \times(A \times A \cup A))^{*}$ associated to the state $v \in \mathcal{A}$ is the set of values of all the paths starting at $v$ and ending at a final state. The language $\mathcal{L}(\mathcal{A})$ associated to $\mathcal{A}$ is the language associated to its initial state. Finally, the language $\mathcal{L}(w)$ associated to $w$ is $\mathcal{L}(w)=$ $\mathcal{L}(\mathcal{A}(w))$.

Note that, if we consider the automaton obtained from $\mathcal{A}(w)$ by replacing the label of each edge in $\mathcal{A}(w)$ by the pair whose first component is the label that this edge has in $\mathcal{A}(w)$ and the second component is the label of the initial vertex of the edge in $\mathcal{A}(w)$, then it is an automaton that recognizes $\mathcal{L}(w)$. We see this replacement in Example 4.12.

Lemma 4.10. Let $\mathcal{A}_{1}$ and $\mathcal{A}_{2}$ be two DA-automata. We have $\mathcal{L}\left(\mathcal{A}_{1}\right)=\mathcal{L}\left(\mathcal{A}_{2}\right)$ if and only if $\overrightarrow{\mathcal{A}_{1}}=\overrightarrow{\mathcal{A}_{2}}$.

Proof. It suffices to note that $\mathcal{L}(\mathcal{A})$ uniquely determines the maximal paths in $\mathcal{A}$, which in turn determines $\overrightarrow{\mathcal{A}}$.

Proposition 4.11. Let $v, w \in \bar{\Omega}_{A} \mathrm{DA}$. Then $\mathrm{DA} \models v=w$ if and only if $\mathcal{L}(v)=$ $\mathcal{L}(w)$.

Proof. By Theorem 4.5, we have $t(v)=t(w)$ if and only if DA $\models v=w$. The result now follows from the previous lemma.

Example 4.12. The wrapped DA-automaton of $w=a^{\omega} b^{\omega} c^{2}(a b)^{\omega} c a b c^{\omega} \in \bar{\Omega}_{A}$ DA is presented in the following figure. Note that it is a finite automaton.

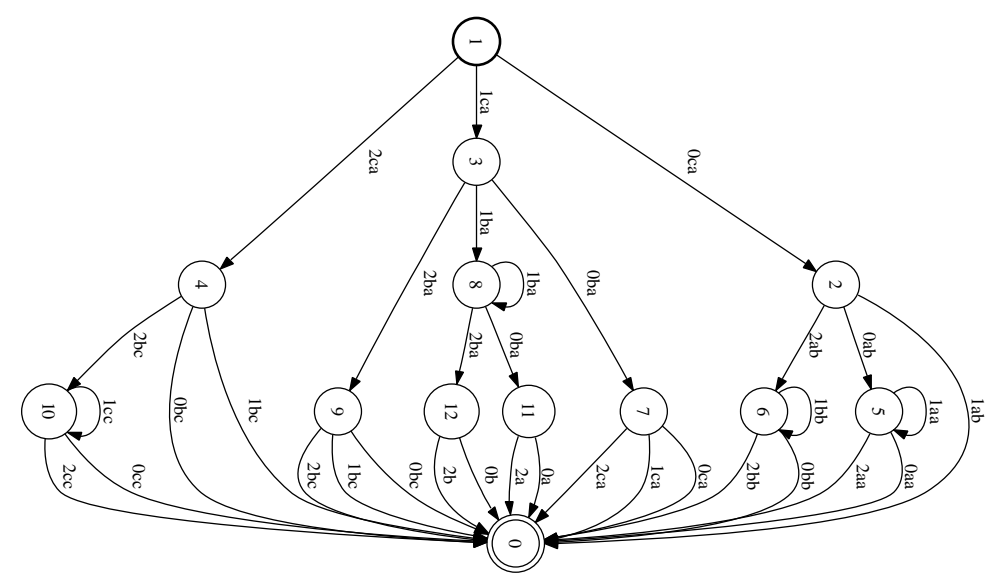


4.2. Representation by labeled orderings. We consider, in the set of linear orderings, the subalgebra generated by the ordinal 1 and by the following operations:

$$
\begin{gathered}
+:\left(o_{1}, o_{2}\right) \mapsto o_{1}+o_{2}, \\
\theta:\left(o_{1}, o_{2}, \ldots, \ldots, o_{2}^{\prime}, o_{1}^{\prime}\right) \mapsto o_{1}+o_{2}+\cdots \cdots+o_{2}^{\prime}+o_{1}^{\prime} .
\end{gathered}
$$

We call an element of this subalgebra a $*$-linear ordering. Note that a $*$-linear ordering has the following properties: it is countable since it is a countable sum of countable orderings, it has a minimum element and a maximum element, any element except the maximum has a successor and any element except the minimum has a predecessor.

Given a $*$-linear ordering $o$, we consider a representation of $o$ in the free algebra generated by $\{1\}$ and by the operations + and $\theta,\langle\{1\} ;+, \theta\rangle$. We define the rank of this representation to be the maximum number of nested operations $\theta$. The rank of $o, \mathfrak{r}(o)$, is the minimum of the ranks of the representations of $o$.

Lemma 4.13. Given a $*$-linear ordering o, any closed interval in o is also a $*$-linear ordering.

Proof. We proceed by induction on the rank of $o$. The case $\mathfrak{r}(o)=0$ is trivial, since it corresponds to the finite linear orderings, and the case $\mathfrak{r}(o)=1$ is easy to show: if $o$ is a $*$-linear ordering such that $\mathfrak{r}(o)=1$, then $o=\left(\omega+\omega^{*}\right) m$, with $m$ finite, and the closed intervals in $o$ are of the form $m$, with $m$ finite, or $\left(\omega+\omega^{*}\right) m^{\prime}$, with $m^{\prime} \leq m$. In any case, they also are $*$-linear orderings. Suppose that the result is verified for $\mathfrak{r}(o)<n$. Let $o$ be a $*$-linear ordering such that $\mathfrak{r}(o)=n>1$. Then $o=\theta\left(o_{1}, o_{2}, \ldots, o_{2}^{\prime}, o_{1}^{\prime}\right) m$ for some representation of $o$, where $o_{i}$ and $o_{i}^{\prime}$ are $*$-linear orderings such that $\mathfrak{r}\left(o_{i}\right)<n$ and $\mathfrak{r}\left(o_{i}^{\prime}\right)<n$, for all $i$, and $m$ is finite. Let $o^{\prime}$ be a closed interval in $o$. Then one of the following cases can occur, for some integers $j$ and $k$ :

(1) $o^{\prime} \subseteq o_{j}$

(2) $o^{\prime} \subseteq o_{k}^{\prime}$;

(3) $o^{\prime} \subseteq o_{j}+o_{j+1}+\cdots+o_{k-1}+o_{k}$ and $o_{j+1}+\cdots+o_{k-1} \subset o^{\prime}$;

(4) $o^{\prime} \subseteq o_{j}^{\prime}+o_{j-1}^{\prime}+\cdots+o_{k+1}^{\prime}+o_{k}^{\prime}$ and $o_{j-1}^{\prime}+\cdots+o_{k+1}^{\prime} \subset o^{\prime}$;

(5) $o^{\prime} \subseteq o_{j}+o_{j+1}+\cdots \cdots+o_{k+1}^{\prime}+o_{k}^{\prime}$ and $o_{j+1}+\cdots \cdots+o_{k+1}^{\prime} \subset o^{\prime}$;

(6) $o^{\prime} \subseteq \bar{o}+\theta\left(o_{1}, o_{2}, \ldots, \ldots, o_{2}^{\prime}, o_{1}^{\prime}\right) m^{\prime}+\bar{o}^{\prime}$ and $\theta\left(o_{1}, o_{2}, \ldots, \ldots, o_{2}^{\prime}, o_{1}^{\prime}\right) m^{\prime} \subset o$, with $0<m^{\prime}<m, \bar{o}$ is of the form (2), (4) or (5) with $k=1$, and $\bar{o}^{\prime}$ is of the form (1), (3) or (5) with $j=1$.

We treat the case (5) in detail. The other cases are similar. In this case, we have that $o_{j} \cap o^{\prime}$ and $o_{k}^{\prime} \cap o^{\prime}$ are closed intervals in $o_{j}$ and $o_{k}^{\prime}$, respectively, and $\mathfrak{r}\left(o_{j}\right)<n$ and $\mathfrak{r}\left(o_{k}^{\prime}\right)<n$. By induction hypothesis, we have that $o_{j} \cap o^{\prime}$ and $o_{k}^{\prime} \cap o^{\prime}$ are *-linear orderings. It follows that $o^{\prime}=\theta\left(o_{j} \cap o^{\prime}, o_{j+1}, \ldots, \ldots, o_{k+1}^{\prime}, o_{k}^{\prime} \cap o^{\prime}\right)$ is a *-linear ordering.

Given a finite alphabet $A$, let $\mathbf{L} \mathbf{O}^{*}(A)$ be the set of $*$-labeled linear orderings in $A$, where an element $(o, l) \in \mathbf{L} \mathbf{O}^{*}(A)$ is such that $o$ is a linear ordering in $\langle\{1\} ;+, \theta\rangle$ and $l$ is a labeling $l: o \rightarrow A$.

Let $\mathbf{o}=(o, l)$ be a $*$-labeled linear ordering. A partition of $\mathbf{o}$ in two non-empty intervals $\left(\mathbf{o}_{\mathbf{1}}, \mathbf{o}_{2}\right)$, where each element of $\mathbf{o}_{\mathbf{2}}$ is greater than all elements of $\mathbf{o}_{\mathbf{1}}$, is called a Dedekind cut in $\mathbf{o}$. We say that a Dedekind cut $\left(\mathbf{o}_{\mathbf{1}}, \mathbf{o}_{\mathbf{2}}\right)$ is a gap in $\mathbf{o}$ if the first interval does not have a maximum and the second interval does not have a minimum. An ordering is complete if it does not have any gap. Given an incomplete 
ordering $\mathbf{o}=(o, l) \in \mathbf{L} \mathbf{O}^{*}(A)$, its completion is isomorphic to the set of Dedekind cuts in o ordered by the relation $\left(\mathbf{o}_{\mathbf{1}}, \mathbf{o}_{\mathbf{2}}\right) \leq\left(\mathbf{o}_{\mathbf{1}}^{\prime}, \mathbf{o}_{\mathbf{2}}^{\prime}\right)$ if $\mathbf{o}_{\mathbf{1}} \subseteq \mathbf{o}_{\mathbf{1}}^{\prime}$. For details, see Rosenstein [12].

Given a Dedekind cut $\left(\mathbf{o}_{1}, \mathbf{o}_{2}\right)$ in $\mathbf{o}$, we define the set of right cofinal letters of $\mathbf{o}_{\mathbf{1}}, c_{r}\left(\mathbf{o}_{\mathbf{1}}\right)$ and the set of left cofinal letters of $\mathbf{o}_{\mathbf{2}}, c_{l}\left(\mathbf{o}_{\mathbf{2}}\right)$ to be the following:

$$
\begin{aligned}
& c_{r}\left(\mathbf{o}_{1}\right)=\left\{a \in A \mid \forall p \in o_{1} \exists q \in o_{1}: p<q \wedge l(q)=a\right\}, \\
& c_{l}\left(\mathbf{o}_{2}\right)=\left\{a \in A \mid \forall p \in o_{2} \exists q \in o_{2}: q<p \wedge l(q)=a\right\} .
\end{aligned}
$$

We say that a $*$-linear ordering o satisfies the cofinal property if, for every Dedekind cut $\left(\mathbf{o}_{1}, \mathbf{o}_{\mathbf{2}}\right)$ in $\mathbf{o}, c_{r}\left(\mathbf{o}_{\mathbf{1}}\right)=c_{l}\left(\mathbf{o}_{\mathbf{2}}\right)$. We call this set the cofinal set of $\left(\mathbf{o}_{\mathbf{1}}, \mathbf{o}_{\mathbf{2}}\right)$ and we write $c\left(\left(\mathbf{o}_{\mathbf{1}}, \mathbf{o}_{\mathbf{2}}\right)\right)=c_{r}\left(\mathbf{o}_{\mathbf{1}}\right)=c_{l}\left(\mathbf{o}_{\mathbf{2}}\right)$.

Let $\mathbf{o} \in \mathbf{L O}^{*}(A)$ be a labeled linear ordering satisfying the cofinal property. We define the following labeling function of the set $\mathcal{D}_{o}$ of Dedekind cuts of $\mathbf{o}$ :

$$
\begin{aligned}
l: \mathcal{D}_{o} & \rightarrow \mathcal{P}(A) \\
\left(\mathbf{o}_{1}, \mathbf{o}_{2}\right) & \mapsto\left\{\begin{array}{cl}
\{a\} & \text { if }\left(\mathbf{o}_{1}, \mathbf{o}_{2}\right) \text { is not a gap and } l\left(\max \mathbf{o}_{\mathbf{1}}\right)=a \\
c\left(\left(\mathbf{o}_{1}, \mathbf{o}_{2}\right)\right) & \text { if }\left(\mathbf{o}_{1}, \mathbf{o}_{2}\right) \text { is a gap. }
\end{array}\right.
\end{aligned}
$$

We say that $\mathbf{o}=(o, l) \in \mathbf{L O}^{*}(A)$ is a reduced A-labeled $*$-linear ordering if it satisfies the following conditions:

(i) o satisfies the cofinal property;

(ii) Given two distinct gaps in o with the same cofinal set, there exists a Dedekind cut between them whose label is not contained in this cofinal set.

Let $\mathbf{r L O}^{*}(A)$ be the set of all reduced $A$-labeled $*$-linear orderings. To each tree $t \in T_{1}(A)$ we associate a $*$-labeled linear ordering $\mu(t)$ by ordering the set of the leaves of $t$ from left to right. Formally, the set of the leaves of $t, F(t)$, is ordered as follows: given two elements $f$ and $f^{\prime}$, let $v_{f f^{\prime}}$ be the deepest node which is a common ancestor of $f$ and $f^{\prime}$, and $v_{f}$ and $v_{f^{\prime}}$ the sons of $v_{f f^{\prime}}$ which are ancestors of $f$ and $f^{\prime}$, respectively (note that we can have $v_{f}=f$ or $v_{f^{\prime}}=f^{\prime}$ ). If $v_{f}<v_{f^{\prime}}$ in the progeny of $v_{f f^{\prime}}$, then we say that $f<f^{\prime}$. It is easy to verify that this defines a linear ordering in $F(t)$. Let $o$ be the corresponding linear ordering and let $l: o \rightarrow A$ be the labeling function which maps each leaf to its label. We put $\mu(t)=(o, l)$.

Proposition 4.14. For each $t \in T_{1}(A), \mu(t) \in \mathbf{r L O}^{*}(A)$.

Proof. We proceed by induction on the height of $t, \mathfrak{h}(t)$, where the case $\mathfrak{h}(t)=0$ is trivial. Recall that the order type of each progeny is $\mathbf{m}$, with $m$ finite, or $\omega+\omega^{*}$. Let $t$ be a tree with non-zero height and let $t_{1}, t_{2}, \ldots, \ldots, t_{2}^{\prime}, t_{1}^{\prime}$ be the subtrees attached to the nodes which are sons of the root of $t$. By induction hypothesis, $o\left(t_{i}\right), o\left(t_{i}^{\prime}\right) \in\langle\{1\} ;+, \theta\rangle$ for all $i$. It follows that $o(t)=o\left(t_{1}\right)+1+o\left(t_{2}\right)+1+$ $\cdots \cdots+1+o\left(t_{2}^{\prime}\right)+1+o\left(t_{1}^{\prime}\right)$ is an element of the subalgebra, since it is a finite or infinite sum of elements of the subalgebra (in the first case, we apply a sufficient number of times the operator + and, in the second case, we apply the operator $\theta)$. Since the height of $t \in T_{1}(A)$ is at most $|A|$, it follows that $\mu(t) \in \mathbf{L} \mathbf{O}^{*}(A)$.

Let $\left(\mathbf{o}_{1}, \mathbf{o}_{2}\right)$ be a Dedekind cut in $\mu(t)$. If this cut is not a gap in $\mu(t)$, then $c_{r}\left(\mathbf{o}_{1}\right)=c_{l}\left(\mathbf{o}_{\mathbf{2}}\right)=\emptyset$. Suppose that it is a gap in $\mu(t)$. This corresponds to dividing the tree in the middle of the progeny of a node $v$ with order $\omega+\omega^{*}$. By Property (4) from the definition of tree in $T_{1}(A)$, we have that $c\left(v_{i}\right) \cup c\left(f_{i}\right)=c\left(v_{i}^{\prime}\right) \cup c\left(f_{i}^{\prime}\right)=c(v)$, for all $i$, where $v_{i}$ and $f_{i}$ are, respectively, the $i$-th node and the $i$-th leaf of the progeny of $v$, when we count from left to right, and $v_{i}^{\prime}$ and $f_{i}^{\prime}$ are, respectively, 
the $i$-th node and the $i$-th leaf of the progeny of $v$, when we count from right to left. Recall that the content of a node is the set of the labels of the descendants leaves. It follows that $c_{r}\left(\mathbf{o}_{1}\right)=c_{l}\left(\mathbf{o}_{2}\right)=c(v)$ and, therefore, $\mu(t)$ satisfies the cofinal property.

Now, we show that condition (ii) holds. Let $\left(\mathbf{o}_{\mathbf{1}}, \mathbf{o}_{\mathbf{2}}\right)<\left(\mathbf{o}_{\mathbf{1}}^{\prime}, \mathbf{o}_{\mathbf{2}}^{\prime}\right)$ be two distinct gaps in $\mathbf{o}$ with the same cofinal set and let $v$ and $v^{\prime}$ be the nodes whose progenies are split in two by the intervals of the gaps $\left(\mathbf{o}_{\mathbf{1}}, \mathbf{o}_{\mathbf{2}}\right)$ and $\left(\mathbf{o}_{\mathbf{1}}^{\prime}, \mathbf{o}_{\mathbf{2}}^{\prime}\right)$, respectively. Let $\bar{v}$ and $\bar{v}^{\prime}$ be the the deepest ancestor of $v$ and $v^{\prime}$, respectively, such that $\bar{v}$ and $\bar{v}^{\prime}$ are in the same progeny. Note that $\bar{v}<\bar{v}^{\prime}$ and that $c\left(\left(\mathbf{o}_{\mathbf{1}}, \mathbf{o}_{\mathbf{2}}\right)\right)=c\left(\left(\mathbf{o}_{\mathbf{1}}^{\prime}, \mathbf{o}_{\mathbf{2}}^{\prime}\right)\right) \subseteq c(\bar{v})$ and $c\left(\left(\mathbf{o}_{\mathbf{1}}, \mathbf{o}_{\mathbf{2}}\right)\right)=c\left(\left(\mathbf{o}_{\mathbf{1}}^{\prime}, \mathbf{o}_{\mathbf{2}}^{\prime}\right)\right) \subseteq c\left(\bar{v}^{\prime}\right)$. Suppose that this progeny has order $\omega+\omega^{*}$. If $\bar{v}$ is in a position of $\omega$, let $f$ be the leaf that succeeds $\bar{v}$ in the progeny and, in case $\bar{v}$ is in a position of $\omega^{*}$, and so is $\bar{v}^{\prime}$, let $f$ be the leaf that precedes $\bar{v}^{\prime}$ in the progeny. Then, by Property (6) from the definition of tree, we have $c(f) \notin c(\bar{v})$ or $c(f) \notin c\left(\bar{v}^{\prime}\right)$. We consider the Dedekind cut in o and between the two gaps, $\left(\mathbf{o}_{\mathbf{1}}^{\prime \prime}, \mathbf{o}_{\mathbf{2}}^{\prime \prime}\right)$, such that $\max o_{1}^{\prime \prime}=f$. It follows that $c\left(\left(\mathbf{o}_{\mathbf{1}}^{\prime \prime}, \mathbf{o}_{\mathbf{2}}^{\prime \prime}\right)\right) \nsubseteq c\left(\left(\mathbf{o}_{\mathbf{1}}, \mathbf{o}_{\mathbf{2}}\right)\right)=c\left(\left(\mathbf{o}_{\mathbf{1}}^{\prime}, \mathbf{o}_{\mathbf{2}}^{\prime}\right)\right)$. The other cases hold similarly. Thus $\mu(t)$ is reduced.

We denote by $\vec{\sum}_{k=1}^{n} o_{k}$ the sum $o_{1}+o_{2}+\cdots+o_{n}$ and by $\overleftarrow{\sum}_{k=1}^{n} o_{k}$ the sum $o_{n}+\cdots+o_{2}+o_{1}$.

Lemma 4.15. Let $t \in T_{1}(A)$ be a tree with non-zero height, let $a_{1}, a_{2}, \ldots, \ldots, b_{2}, b_{1}$ be the labels of the leaves that are sons of the root and let $t_{1}, t_{2}, \ldots, \ldots, t_{2}^{\prime}, t_{1}^{\prime}$ be the subtrees of $t$ attached to each son of the root. If $\mu\left(t_{m}\right)=\left(o_{m}, l_{m}\right)$ and $\mu\left(t_{m}^{\prime}\right)=$ $\left(o_{m}^{\prime}, l_{m}^{\prime}\right)$, for all $m$, then $\mu(t)=(o, l)$ where o is a*-linear ordering of one of the following forms:

(1) $\vec{\sum}_{n \geq 1}\left(o_{n}+1\right)+\overleftarrow{\sum}_{n \geq 1}\left(1+o_{n}^{\prime}\right)$, if the leaf sons have order $\omega+\omega^{*}$

(2) $\vec{\sum}_{i=1}^{n}\left(o_{i}+1\right)+o_{n+1}+\overleftarrow{\sum}_{i=1}^{n}\left(1+o_{i}^{\prime}\right)$, if the number of leaf sons is even;

(3) $\stackrel{\vec{\sum}}{i=1}_{n}^{n}\left(o_{i}+1\right)+o_{n}^{\prime}+\overleftarrow{\sum}_{i=1}^{n-1}\left(1+o_{i}^{\prime}\right)$, if the number of leaf sons is odd; and $l$ is the labeling $l: o \rightarrow A$ satisfying the following conditions:

(i) $l\left(\vec{\sum}_{i=1}^{m-1}\left(o_{i}+1\right)+\gamma\right)=l_{m}(\gamma)$, if $\gamma$ is an initial segment of $o_{m}$,

(ii) $l\left(\vec{\sum}_{i \geq 1}\left(o_{i}+1\right)+\overleftarrow{\sum}_{i>m}\left(1+o_{i}^{\prime}\right)+1+\gamma\right)=l_{m}^{\prime}(\gamma)$, if $\gamma$ is an initial segment of $o_{m}^{\prime}$

(iii) $l\left(\vec{\sum}_{i=1}^{m}\left(o_{i}+1\right)\right)=a_{m}$,

(iv) $l\left(\vec{\sum}_{i \geq 1}\left(o_{i}+1\right)+\overleftarrow{\sum}_{i>m}\left(1+o_{i}^{\prime}\right)+1\right)=b_{m}$

Proof. The verification follows directly by induction on the height of $t$.

To establish that $\mu$ is a bijection, we construct and iterate a central basic partition of a non-zero reduced $*$-labeled linear ordering. We start with the following lemma.

Lemma 4.16. Let $\mathbf{o}=(o, l) \in \mathbf{r} \mathbf{L O} \mathbf{O}^{*}(A)$. Then, for each $a \in c(\mathbf{o})$, there exist the smallest position of $\mathbf{o}$ labeled $a$ and the largest position of $\mathbf{o}$ labeled $a$.

Proof. For each $a \in c(\mathbf{o})$, let $D_{a}=\left\{\left(\mathbf{o}_{1}, \mathbf{o}_{2}\right) \in \mathcal{D}_{o} \mid a \in l\left(\left(\mathbf{o}_{1}, \mathbf{o}_{2}\right)\right)\right\}$. This subset is non-empty and bounded below, so, since $\mathcal{D}_{o}$ is a complete ordering, it has an infimum, $\left(\mathbf{o}_{1}, \mathbf{o}_{2}\right)$. Suppose that it is a gap in o. By definition of infimum, for every Dedekind cut $\left(\mathbf{o}_{\mathbf{1}}^{\prime}, \mathbf{o}_{\mathbf{2}}^{\prime}\right)>\left(\mathbf{o}_{\mathbf{1}}, \mathbf{o}_{\mathbf{2}}\right)$, there exists a Dedekind cut $\left(\mathbf{o}_{\mathbf{1}}^{\prime \prime}, \mathbf{o}_{\mathbf{2}}^{\prime \prime}\right)$ such 
that $\left(\mathbf{o}_{\mathbf{1}}, \mathbf{o}_{\mathbf{2}}\right) \leq\left(\mathbf{o}_{\mathbf{1}}^{\prime \prime}, \mathbf{o}_{\mathbf{2}}^{\prime \prime}\right)<\left(\mathbf{o}_{\mathbf{1}}^{\prime}, \mathbf{o}_{\mathbf{2}}^{\prime}\right)$ and $a \in l\left(\left(\mathbf{o}_{\mathbf{1}}^{\prime \prime}, \mathbf{o}_{\mathbf{2}}^{\prime \prime}\right)\right)$. But this is equivalent to $a \in c\left(\left(\mathbf{o}_{1}, \mathbf{o}_{2}\right)\right)$ and, therefore, there exists a sequence of Dedekind cuts less than $\left(\mathbf{o}_{\mathbf{1}}, \mathbf{o}_{\mathbf{2}}\right)$ whose label contains $a$, which is contrary to the hypothesis of $\left(\mathbf{o}_{\mathbf{1}}, \mathbf{o}_{\mathbf{2}}\right)$ be an infimum. Thus $\left(\mathbf{o}_{\mathbf{1}}, \mathbf{o}_{\mathbf{2}}\right)$ is not a gap and, therefore, it is minimum for, otherwise, its successor will be also a lower bound of $D_{a}$. Since $\left(\mathbf{o}_{\mathbf{1}}, \mathbf{o}_{\mathbf{2}}\right)$ is not a gap, $\mathbf{o}_{\mathbf{1}}$ has a maximum and $\mathbf{o}_{\mathbf{2}}$ has a minimum. The maximum of $\mathbf{o}_{\mathbf{1}}$ is the smallest position of $\mathbf{o}$ labeled $a$. Similarly, we prove the existence of the largest position of o labeled $a$.

Let $\mathbf{o}=(o, l) \in \mathbf{r L O}^{*}(A)$. For each letter $a \in A$, let $p_{a}^{o}$ be the smallest position of o such that $l(p)=a$, let $\bar{p}_{a}^{o}$ be the largest position of $\mathbf{o}$ such that $l(p)=a$ and let $p^{o}=\max \left\{p_{a}^{o} \mid a \in A\right\}$ and $\bar{p}^{o}=\min \left\{\bar{p}_{a}^{o} \mid a \in A\right\}$ (if there is no position labeled by $a$, we set $p_{a}^{o}=\min o$ and $\bar{p}_{a}^{o}=\max o$ ). Note that, since $A$ is finite, there exist $p^{o}$ and $\bar{p}^{o}$. Three cases can occur: $p^{o}<\bar{p}^{o}, p^{o}>\bar{p}^{o}$ or $p^{o}=\bar{p}^{o}$. We begin with the case $p^{o}<\bar{p}^{o}$. Let $\alpha_{1}=\left[\min o, p^{o}\left[, \gamma_{1}=\right] p^{o}, \bar{p}^{o}\left[\right.\right.$ and $\left.\beta_{1}=\right] \bar{p}^{o}$, max $\left.o\right]$, which are also $*$-linear orderings by Lemma 4.13, since they are closed intervals in $o$. We have $o=\alpha_{1}+1+\gamma_{1}+1+\beta_{1}$. We call this equality the central basic partition of $(o, l)$. Let $\gamma_{0}=o, p_{1}=p^{o}$ and $\bar{p}_{1}=\bar{p}^{o}$. While $p_{i}<\bar{p}_{i}$ and $c\left(\gamma_{i}\right)=c(o)$, let $p_{i+1}=$ $\max \left\{p_{a}^{o} \in \gamma_{i} \mid a \in A\right\}, \bar{p}_{i+1}=\min \left\{\bar{p}_{a}^{o} \in \gamma_{i} \mid a \in A\right\}$ and $\alpha_{i+1}=\left[\min \gamma_{i}, p_{i+1}[\right.$, $\left.\gamma_{i+1}=\right] p_{i+1}, \bar{p}_{i+1}\left[\right.$ and $\left.\left.\beta_{i+1}=\right] \bar{p}_{i+1}, \max \gamma_{i}\right]$ be the $*$-linear orderings such that $\gamma_{i}=\alpha_{i+1}+1+\gamma_{i+1}+1+\beta_{i+1}$. If, for any $k, c\left(\gamma_{k+1}\right) \neq c(o)$, or $p_{k}>\bar{p}_{k}$, or $p_{k}=\bar{p}_{k}$, then we put, respectively:

(i) $\gamma_{k}=\alpha_{k+1}+1+\gamma_{k+1}+1+\beta_{k+1}$, where $\alpha_{k+1}=\left[\min \gamma_{k}, p_{k+1}\left[, \gamma_{k+1}=\right.\right.$ ]$p_{k+1}, \bar{p}_{k+1}\left[\right.$ and $\left.\left.\beta_{k+1}=\right] \bar{p}_{k+1}, \max \gamma_{k}\right]$,

(ii) $\gamma_{k}=\alpha_{k+1}+1+\gamma_{k+1}+1+\beta_{k+1}$, where $\alpha_{k+1}=\left[\min \gamma_{k}, \bar{p}_{k+1}\left[, \gamma_{k+1}=\right.\right.$ ] $\bar{p}_{k+1}, p_{k+1}\left[\right.$, and $\left.\left.\beta_{k+1}=\right] p_{k+1}, \max \gamma_{k}\right]$,

(iii) $\gamma_{k}=\alpha_{k+1}+1+\beta_{k+1}$ where $\alpha_{k+1}=\left[\min \gamma_{k}, p_{k}\left[=\left[\min \gamma_{k}, \bar{p}_{k}\left[\right.\right.\right.\right.$ and $\beta_{k+1}=$ ]$\left.\left.\left.p_{k}, \max \gamma_{k}\right]=\right] \bar{p}_{k}, \max \gamma_{k}\right]$.

In these cases, we stop the iteration and we obtain one of the following equalities:

$$
o=\vec{\sum}_{i=1}^{k+1}\left(\alpha_{i}+1\right)+\gamma_{k+1}+\overleftarrow{\sum}_{i=1}^{k+1}\left(1+\beta_{i}\right)
$$

if $c\left(\gamma_{k+1}\right) \neq c(o)$ or $p_{k}>\bar{p}_{k}$, or

$$
o=\vec{\sum}_{i=1}^{k+1}\left(\alpha_{i}+1\right)+\beta_{k+1}+\overleftarrow{\sum}_{i=1}^{k}\left(1+\beta_{i}\right)
$$

if $p_{k}=\bar{p}_{k}$. Let $l_{0}=l$. For each $i$, let $l_{i}^{\prime}$ be the restriction of $l_{i-1}$ to the initial segment $\alpha_{i}$ of $\gamma_{i-1}, l_{i}^{\prime \prime}$ be the labeling of $\beta_{i}$ defined by $l_{i}^{\prime \prime}(\delta)=l_{i-1}\left(\alpha_{i}+1+\gamma_{i}+1+\delta\right)$, where $\delta$ is an initial segment of $\beta_{i}$, and $l_{i}$ be the labeling of $\gamma_{i}$ defined by $l_{i}(\delta)=l_{i-1}\left(\alpha_{i}+1+\delta\right)$, where $\delta$ is an initial segment of $\gamma_{i}$. For each $m \geq 1$, we obtain

(i) $l_{m}^{\prime}(\delta)=l\left(\vec{\sum}_{i=1}^{m-1}\left(\alpha_{i}+1\right)+\delta\right)$, if $\delta$ is an initial segment of $\alpha_{m}$,

(ii) $l_{m}(\delta)=l\left(\sum_{i=1}^{m}\left(\alpha_{i}+1\right)+\delta\right)$, if $\delta$ is an initial segment of $\gamma_{m}$,

(iii) $l_{m}^{\prime \prime}(\delta)=l\left(\sum_{i=1}^{m}\left(\alpha_{i}+1\right)+\gamma_{m}+1+\delta\right)$, if $\delta$ is an initial segment of $\beta_{m}$.

This defines the iterated central basic partition of $\mathbf{o}$.

In case $p_{i}^{o}<\bar{p}_{i}^{o}$ and $c\left(\gamma_{i}\right)=c(o)$, for all $i$, we iterate indefinitely the partition defined above and we obtain $o=\vec{\sum}_{i \geq 1}\left(\alpha_{i}+1\right)+\overleftarrow{\sum}_{i \geq 1}\left(1+\beta_{i}\right)$ as justified by the following lemma. 
Lemma 4.17. Let $\mathbf{o}=(o, l) \in \mathbf{r L O} \mathbf{O}^{*}(A)$. If $\mathbf{o}$ has an infinite iterated central basic partition, then $o=\vec{\sum}_{i \geq 1}\left(\alpha_{i}+1\right)+\overleftarrow{\sum}_{i \geq 1}\left(1+\beta_{i}\right)$

Proof. Let $P=\left\{p_{i}^{o}: i \geq 1\right\}$ and $\bar{P}=\left\{\bar{p}_{i}^{o}: i \geq 1\right\}$. These sets are infinite, $P$ is bounded above and it does not have a maximum element and $\bar{P}$ is bounded below and it does not have a minimum element. We consider the subsets of $\mathcal{D}_{o}$ that follow:

$$
\begin{aligned}
& D=\left\{\left(\mathbf{o}_{1}, \mathbf{o}_{2}\right) \in \mathcal{D}_{o} \mid \exists p_{i}^{o} \in P, p_{i}^{o}=\max o_{1}\right\}, \\
& \bar{D}=\left\{\left(\mathbf{o}_{1}, \mathbf{o}_{2}\right) \in \mathcal{D}_{o} \mid \exists \bar{p}_{i}^{o} \in \bar{P}, \bar{p}_{i}^{o}=\max o_{1}\right\} .
\end{aligned}
$$

Let $\left(\mathbf{o}_{\mathbf{1}}, \mathbf{o}_{\mathbf{2}}\right)=\sup D$ and $\left(\mathbf{o}_{\mathbf{1}}^{\prime}, \mathbf{o}_{\mathbf{2}}^{\prime}\right)=\inf \bar{D}$, which exist, since $\mathcal{D}_{o}$ is a complete ordering, $D$ is bounded above and $\bar{D}$ is bounded below. Note that these Dedekind cuts are gaps in o, since $D$ does not have a maximum and $\bar{D}$ does not have a minimum. Moreover, by definition of iterated central basic partition of an ordering $\mathbf{o}$ and by definition of cofinal set of a gap in $\mathbf{o}$, we have $c\left(\left(\mathbf{o}_{\mathbf{1}}, \mathbf{o}_{\mathbf{2}}\right)\right)=c\left(\left(\mathbf{o}_{\mathbf{1}}^{\prime}, \mathbf{o}_{\mathbf{2}}^{\prime}\right)\right)=$ $c(\mathbf{o})$. If these gaps are distinct, then, by definition of reduced $*$-labeled linear ordering, there exists a Dedekind cut between them whose label does not belong to $c(\mathbf{o})$, which is a contradiction. It follows that $\left(\mathbf{o}_{\mathbf{1}}, \mathbf{o}_{\mathbf{2}}\right)=\left(\mathbf{o}_{\mathbf{1}}^{\prime}, \mathbf{o}_{\mathbf{2}}^{\prime}\right)$ and, therefore, $o=\vec{\sum}_{i \geq 1}\left(\alpha_{i}+1\right)+\overleftarrow{\sum}_{i \geq 1}\left(1+\beta_{i}\right)$

Let $\nu: \mathbf{r L O}^{*}(A) \rightarrow T_{1}(A)$ be the mapping defined as follows. If $o=0$, then $\nu(o, l)$ is the tree which consists of a unique degenerate node. If $o \neq 0$, then we consider the iterated central basic partition of $(o, l), o=\vec{\sum}_{i \geq 1}\left(\alpha_{i}+1\right)+\overleftarrow{\sum}_{i \geq 1}\left(1+\beta_{i}\right)$ Note that $c\left(\alpha_{i}, l_{i}^{\prime}\right)$ and $c\left(\beta_{i}, l_{i}^{\prime \prime}\right)$ are strictly contained in $c(o, l)$, for all $i$. We defined the tree $\nu(o, l)$ by induction on the content of $(o, l)$ : to each element of the sum $o=\vec{\sum}_{i \geq 1}\left(\alpha_{i}+1\right)+\overleftarrow{\sum}_{i \geq 1}\left(1+\beta_{i}\right)$ corresponds a son of the root. The vertex which corresponds to an element $\alpha_{i}$ or $\beta_{i}$, for all $i$ (and also to $\gamma_{k}$, if it exists) is a node whose content is the image of the labelings $l_{i}^{\prime}$ and $l_{i}^{\prime \prime}$, respectively (if $\gamma_{k}$ exists, then the content of the corresponding vertex is the image of $l_{k}$ ). We note that, if one of those orderings is 0 , the corresponding vertex is a degenerate node. To the rest of the elements of the sum we associate a leaf labeled, respectively, $l\left(\alpha_{1}+1\right), l\left(\alpha_{1}+1+\alpha_{2}+1\right), \ldots, l\left(\alpha_{1}+1+\alpha_{2}+1+\cdots \cdots+1+\beta_{3}+1\right)$, $l\left(\alpha_{1}+1+\alpha_{2}+1+\cdots \cdots+1+\beta_{3}+1+\beta_{2}+1\right)$. We attach to the vertices which do not correspond to a degenerate node the subtrees $\nu\left(\alpha_{1}, l_{1}^{\prime}\right), \nu\left(\alpha_{2}, l_{2}^{\prime}\right), \ldots$, $\ldots, \nu\left(\beta_{2}, l_{2}^{\prime \prime}\right), \nu\left(\beta_{1}, l_{1}^{\prime \prime}\right)$. We notice that the trees constructed in this manner are, effectively, in $T_{1}(A)$. In fact, we can verified immediately the properties (1)-(5) from the definition of tree in $T_{1}(A)$ in the construction made. The properties (6)(8) follow by the definition of iterated central basic factorization of $(o, l)$ and the fact that the orderings involved are reduced.

Theorem 4.18. The mapping $\mu: T_{1}(A) \rightarrow \mathbf{r L O}^{*}(A)$ is a bijection.

Proof. Let $t \in T(A)$. If $\mathfrak{h}(t)=0$, then $\nu(\mu(t))=t$ by definition. Suppose that $t$ has non-zero height and let $\mu(t)=(o, l)$. By Lemma 4.15 and by definition of iterated central basic partition of a reduced $*$-labeled linear ordering, we have that $o=\vec{\sum}_{n \geq 1}\left(o_{n}+1\right)+\sum_{n \geq 1}\left(1+o_{n}^{\prime}\right)$ is exactly the iterated central basic partition of $(o, l)$ and, therefore, $\nu(\mu(t))=t$. Now, let $(o, l) \in \mathbf{r L O}^{*}(A)$. The case $o=0$ follows by definition. Suppose that $o \neq 0$ and let $o=\vec{\sum}_{i \geq 1}\left(\alpha_{i}+1\right)+\overleftarrow{\sum}_{i \geq 1}\left(1+\beta_{i}\right)$ be the iterated central basic partition of $o=(o, l)$. For each $i$, let $l_{\alpha_{i}}: \alpha_{i} \rightarrow A$ be defined by $l_{\alpha_{i}}(\delta)=l_{i}^{\prime}(\delta)$, where $\delta$ is an initial segment of $\alpha_{i}$, and $l_{\beta_{i}}: \beta_{i} \rightarrow A$ 
is defined by $l_{\beta_{i}}(\delta)=l_{i}^{\prime \prime}(\delta)$, where $\delta$ is an initial segment of $\beta_{i}$. Consider the tree whose leaf sons of the root are labeled $l\left(\alpha_{1}+1\right), l\left(\alpha_{1}+1+\alpha_{2}+1\right), \ldots$, $l\left(\alpha_{1}+1+\alpha_{2}+1+\cdots \cdots+1+\beta_{3}+1\right), l\left(\alpha_{1}+1+\alpha_{2}+1+\cdots \cdots+1+\beta_{3}+1+\beta_{2}+1\right)$ and whose subtrees attached to the nodes of the progeny of the root are $\nu\left(\alpha_{1}, l_{\alpha_{1}}\right)$, $\nu\left(\alpha_{2}, l_{\alpha_{2}}\right), \ldots, \nu\left(\beta_{2}, l_{\beta_{2}}\right), \nu\left(\beta_{1}, l_{\beta_{1}}\right)$. It follows, by Lemma 4.15 , that $\mu(\nu(o, l))=$ $(o, l)$.

We finish by showing the relation between the representations of implicit operations by finite-height trees and by labeled orderings. It will be useful to relate also these representations with the representation by quasi-ternary trees. In fact, we can construct a bijection $\xi: T_{2}(A) \rightarrow T_{1}(A)$ recursively as follows. Let $t \in T_{2}(A)$ be such that $\rho(t)=\rho\left(t_{0}\right) \cdot l_{(\varepsilon, 0)} \cdot \rho\left(t_{10}\right) \cdot l_{(1,0)} \cdots \cdots l_{(1,2)} \cdot \rho\left(t_{12}\right) \cdot l_{(\varepsilon, 2)} \cdot \rho\left(t_{2}\right)$, where $\rho: T_{2}(A) \rightarrow \bar{\Omega}_{A}$ DA is the bijection defined in 4.1.2. Let $i$ be maximum for $c\left(v_{1^{i}}\right)=$ $c(\rho(t))$, where $v_{1^{i}}$ is the root of the subtree $t_{1^{i}}$. Then the progeny of the root of $\xi(t)$ consists of the leaves labeled by $l_{(\epsilon, 0)}, l_{(1,0)}, \ldots, l_{\left(1^{i}, 0\right)}, l_{\left(1^{i}, 2\right)}, \ldots, l_{(1,2)}, l_{(\epsilon, 0)}$ and the trees $\rho\left(t_{0}\right), \rho\left(t_{10}\right), \ldots, \rho\left(t_{1^{i} 0}\right), \rho\left(t_{1^{i+1}}\right), \rho\left(t_{1^{i} 2}\right), \ldots, \rho\left(t_{12}\right), \rho\left(t_{2}\right)$ attached to the nodes. Proceeding recursively, and since $A$ is finite, we obtain the tree $\xi(t) \in T_{1}(A)$. We leave the details to the reader.

\section{ACKNOWLEDGMENTS}

This work is part of the author's doctoral thesis, written under the supervision of Prof. Jorge Almeida, from whose advice the author has greatly benefited. This work was supported by the Fundação para a Ciência e a Tecnologia (FCT) through the PhD grant SFRH/BD/19720/2004, through the Centro de Matemática da Universidade do Porto (CMUP) and also through the project PTDC/MAT/65481/2006, which is partly funded by the European Community Fund FEDER.

\section{REFERENCES}

[1] J. Almeida, Implicit operations on finite J-trivial semigroups and a conjecture of I. Simon, J. Pure and Applied Algebra 69 (1990) 205-218.

[2] , Finite Semigroups and Universal Algebra, World Scientific, Singapore, 1994. English translation.

[3] - A syntactical proof of locality of DA, Int. J. Algebra and Comp. 6 (1996) 165-177.

[4] - Finite semigroups: an introduction to a unified theory of pseudovarieties, in Semigroups, Algorithms, Automata and Languages, J.-E. P. G. M. S. Gomes and P. V. Silva, eds., Singapore, 2002, World Scientific, 3-64.

[5] J. Almeida, J. C. Costa, and Zeitoun, Iterated periodicity over finite aperiodic semigroups, Tech. Rep. CMUP 2009-03, Univ. of Porto, 2009.

[6] J. Almeida, J. C. Costa, and M. Zeitoun, Some structural properties of the free profinite aperiodic semigroup, in Proc. of the conference "AutoMathA: from Mathematics to Applications", Univ. Liège, June 2009, 2009.

[7] J. Almeida and P. Weil, Free profinite R-trivial monoids, Int. J. Algebra and Comput. 7 (1997) 625-671.

[8] J. Almeida and M. Zeitoun, An automata-theoretic approach to the word problem for $\omega$-terms over R, Theor. Comp. Sci. 370 (2007) 131-169.

[9] A. Azevedo, Operations preserving homomorphisms on the class of finite semigroups DS, in Actas II Encontro de Algebristas Portugueses, J. Almeida, ed., Porto, 1987, Universidade do Porto, 33-43.

[10] S. Eilenberg, Automata, Languages and Machines, vol. B, Academic Press, New York, 1976.

[11] J. Reiterman, The Birkhoff theorem for finite algebras, Algebra Universalis 14 (1982) 1-10.

[12] J. G. Rosenstein, Linear Orderings, Academic Press, New York, 1982. 
[13] M. P. Schützenberger, Sur le produit de concaténation non ambigu, Semigroup Forum 13 (1976) 47-75.

[14] P. Tesson and D. Thérien, Diamonds are forever: the variety DA, in Semigroups, Algorithms, Automata and Languages, World Scientific, 2002, 475-499.

[15] P. Trotter and P. Weil, The lattice of pseudovarieties of idempotent semigroups and a nonregular analogue, Algebra Univers. 37 (1997) 491-526.

Instituto Superior de Engenharia do Porto/LEMA and Centro de Matemática da Universidade do Porto, Rua do Campo Alegre, 687, 4169-007 Porto, Portugal

E-mail address: aim@isep.ipp.pt/amoura@fc.up.pt 